\title{
UNIONES DE CADENAS EN ESPACIOS DIÁDICOS Y GRUPOS TOPOLÓGICOS
}

Tesis que presenta la

M en C. YOLANDA MAGDA TORRES FALCÓN para la obtención del grado de DOCTORA EN CIENCIAS

JUNIO 2001

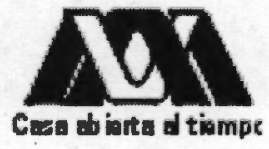

UNIVERSIDAD AUTÓNOMA METROPOLTANA-IZTAPALAPA DIVISTÓN DE CIENCIAS BÁSICAS E INGENIERÍA 
Para Philip

Para mis hijos, Charly y Alex

When forty winters shall besiege thy brow, And dig deep trenches in thy beauty's field, Thy youth's proud livery, so gaz d on now, Will be a tatter' d weed, of small worth held: Then being ask $d$ where all thy beauty lies, Where all the treasure of thy lusty days;

To say, within thine own deep sunken eyes, Were an all-eating shame and thriftless praise.

How much more praise deserv'd thy beauty's use, If thou couldst answer - "This fair child of mine Shall sum my count, and make my old excuse-' Proving his beauty by succession thine!

This were to be new-made when thou art old, And see thy blood warm when thou feel st it cold.

William Shakespeare 


\section{Agradecimientos}

Quiero expresar mi más profunda gratitud al Dr. Mikhail Tkachenko por su excelente labor en la dirección de este trabajo. Durante todo este tiempo su apoyo, su atención a mi trabajo y su gran generosidad, tanto con su tiempo como con sus conocimientos, han sido constantes en mi vida. Él ha sido para mí mucho más que un maravilloso maestro: un ejemplo, una inspiración y un gran amigo.

También quiero agradecer al Dr. Vladimir Tkachuk, en cuyo seminario he aprendido mucho y quien me permitió, durante la ausencia de Mikhail, estar en la privilegiada situación de tener todos los derechos de sus alumnos y ninguna de sus obligaciones.

Mi amigo, el Dr. Constancio Hernández, me enseñó todo lo que sé de $\mathrm{BTT}_{\mathrm{EX}}$ y me ayudó constantemente en la preparación de este manuscrito. Muchas gracias.

Finalmente, agradezco a los sinodales: Dr. Oleg Okunev, Dr. Angel Tamariz, Dr. Mikhail Tkachenko, Dr. Vladimir Tkachuk y Dr. Richard Wilson, por la cuidadosa lectura que hicieron de esta tesis y por sus comentarios, que me permitieron mejorarla. 


\section{Índice}

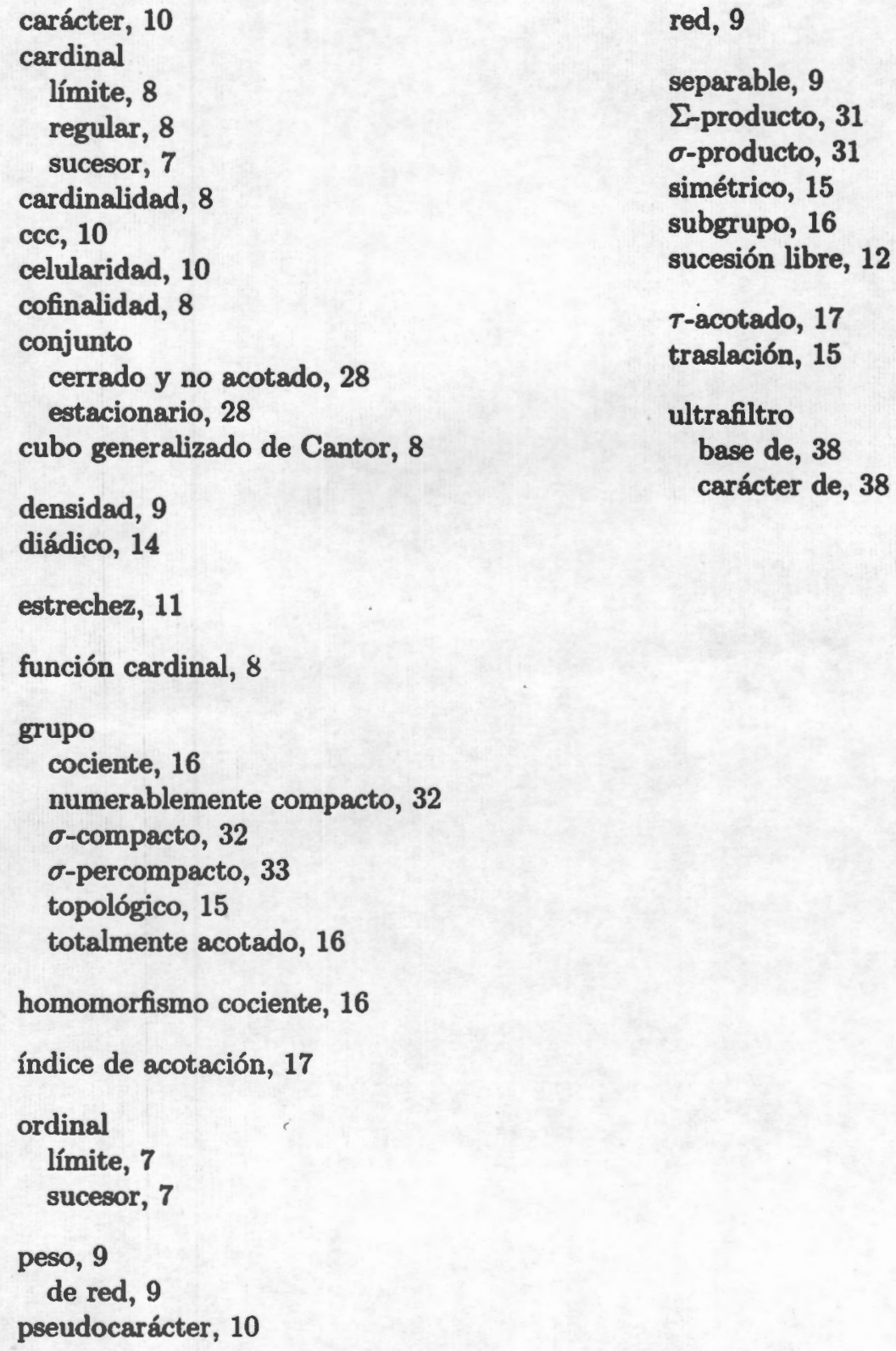

red, 9

separable, 9

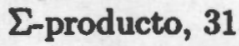

$\sigma$-producto, 31

simétrico, 15

subgrupo, 16

sucesión libre, 12

$\tau$-acotado, 17

traslación, 15

ultrafiltro

base de, 38

carácter de, 38 


\section{Contenido}

$\begin{array}{ll}\text { Introducción } & 1\end{array}$

CAPÍTULO 1. Preliminares : 7

$\begin{array}{ll}\text { 1. Notación y definiciones } & 7\end{array}$

2. Funciones cardinales 8

3. Cubos generalizados de Cantor 12

4. Grupos topológicos 15

5. Uniones de cadenas $\quad 19$

CAPÍTULO 2. Espacios diádicos y grupos localmente compactos 25

1. Pseudocarácter y estrechez 25

2. Algunos ejemplos 31

3. Grupos totalmente acotados y $\sigma$-compactos 33

4. Observaciones finales $\quad 34$

CAPÍtulo 3. Cadenas de subgrupos $\quad 35$

1. Introducción 35

2. Indice de acotación $\quad 35$

3. Cotas para la cardinalidad de los grupos 39

$\begin{array}{ll}\text { Bibliografia } & 45\end{array}$

$\begin{array}{ll}\text { Índice analitico } & 47\end{array}$ 


\section{Introducción}

La topología y la teoría de conjuntos, a pesar de tener un origen común, se desarrollaron en direcciones distintas durante mucho tiempo. Un evento cambió este estado de cosas: en los años 60 Paul Cohen demostró que la negación de la hipótesis del continuo también es consistente con los axiomas usuales de la teoría de conjuntos. El teorema en sí no es de mucho interés para la topología, lo interesante es la técnica empleada en su demostración, llamada forcing. Esta técnica puede ser traducida en términos de álgebras booleanas, en términos de órdenes parciales y en términos que generan enunciados combinatorios aplicables a una gran variedad de problemas relacionados con espacios topológicos abstractos.

A finales de la década de los 60 , había gran actividad en la topología de conjuntos: había muchas preguntas topológicas de carácter conjuntista, existía la posibilidad de utilizar técnicas combinatorias y nuevas traducciones de las técnicas de forcing y en algunos casos los teóricos de conjuntos lograron solucionar algunos problemas topológicos. El manejo de herramientas de la teoría de conjuntos para la solución de problemas topológicos hizo posible una interacción muy fructífera entre estas dos disciplinas que continúa hasta hoy en día.

Claramente no todos los problemas topológicos admiten una traducción en términos de conjuntos. Tenemos que identificar hasta qué punto un problema topológico es de carácter conjuntista, es decir, hasta qué punto se puede traducir en términos de aritmética cardinal. La teoría de funciones cardinales en topología se encarga precisamente de esto.

Las funciones cardinales permiten extender propiedades topológicas importantes, como por ejemplo la de tener una base numerable, ser separable o ser primero numerable, a cardinalidades más grandes. Una función cardinal asigna a cada espacio topológico $X$, un cardinal infinito. La petición de que los valores de las funciones cardinales sean infinitos simplifica los enunciados de muchos resultados y pone énfasis en la aritmética cardinal infinita.

La función cardinal más obvia es la cardinalidad del espacio $X$ (más $\omega$ ), denotada por $|X|$; una función cardinal mucho más útil (quizás la más útil) es el peso de $X$, que es la mínima cardinalidad de una base para $X$ (más $\omega$ ). Un espacio segundo numerable tiene peso $\omega$. Una red para un espacio topológico $X$ es una familia $\mathcal{R}$ de subconjuntos de $X$ tal que, para todo punto $p \in X$ 
y todo abierto $U$ de $X$ tal que $p \in U$, existe $R \in \mathcal{R}$ con $p \in R \subseteq U$. El peso de red de $X$ es la mínima cardinalidad de una red para $X$ (más $\omega$ ). El carácter de $X$ en el punto $p$ es la mínima cardinalidad de una base local en $p$ y se denota $\chi(p, X)$; tomando el supremo de todos estos cardinales obtenemos el carácter de $X$, es decir, $\chi(X)=\sup \{\chi(p, X): p \in X\}+\omega$. Los espacios primero numerables tienen carácter $\omega$. La densidad de un espacio $X$ es la mínima cardinalidad de un subconjunto denso de $X$ (más $\omega$ ). Los espacios separables tienen densidad $\omega$. El número de Lindelöf del espacio $X$, denotado $L(X)$, es el mínimo cardinal infinito $\alpha$ tal que toda cubierta abierta de $X$ tiene una subcubierta de cardinalidad $\leq \alpha$. Los espacios compactos y los espacios de Lindelöf tienen número de Lindelöf $\omega$. El pseudocarácter de un espacio $X$ en un punto $p$, en símbolos $\psi(p, X)$, es la mínima cardinalidad de una familia de abiertos $U$ tal que $\cap \mathcal{U}=\{p\}$. El pseudocarácter de $X$ se define como $\psi(X)=\sup \{\psi(p, X): p \in X\}+\omega$. La estrechez de un espacio topológico $X$ en un punto $p$, denotada por $t(p, X)$, es el mínimo cardinal $\kappa$ tal que para todo $Y \subseteq X$ con $p \in \bar{Y}$, existe $A \subseteq Y$ tal que $|A| \leq \kappa$ y $p \in \bar{A}$. La estrechez del espacio $X$ es entonces $t(X)=\sup \{t(p, X): p \in X\}+\omega$. Finalmente, si $\phi$ es una función cardinal, la función $\phi$ hereditaria se obtiene tomando el supremo de los valores de $\phi$ en todos los subespacios de $X$, es decir, $h \phi(X)=\sup \{\phi(M): M \subseteq X\}$. Muchas propiedades topológicas corresponden a la hipótesis de que alguna función cardinal natural sea numerable.

Aunque el estudio sistemático de las funciones cardinales no empezó sino hasta la década de los 60 , muchos resultados aislados se obtuvieron mucho antes.

En los años 20 Alexandroff y Urysohn desarrollaron la teoría básica de los espacios compactos. Un resultado obtenido durante ese tiempo afirma que un espacio compacto y perfectamente normal tiene cardinalidad $\leq 2^{\omega}$. Alexandroff y Urysohn dejaron abierta la pregunta de si todo espacio compacto primero numerable tiene cardinalidad a lo sumo $2^{\omega}$.

En la década de los 40 Hewitt, Marczewski y Pondiczery demostraron un teorema sorprendente acerca de la densidad en productos de espacios. Probaron que el producto de una familia de espacios, todos con densidad $\leq \alpha$ tiene densidad $\leq \alpha$, siempre y cuando la cardinalidad de la familia no exceda $2^{\alpha}$. En su versión numerable este teorema establece que el producto de a lo sumo $2^{\omega}$ espacios separables es separable.

A partir de 1960 el estudio de las funciones cardinales se inició en forma sistemática, de Groot en 1965 publicó un artículo en el que introdujo nuevas funciones cardinales importantes y demostró que todo espacio de Hausdorff tal que todos sus subespacios son de Lindelöf tiene cardinalidad a lo sumo $2^{\omega}$, generalizando el resultado previo de que todo espacio compacto perfectamente normal tiene cardinalidad a lo sumo $2^{\omega}$. 
Más tarde Hajnal y Juhász extendieron el trabajo realizado por de Groot y entre otros resultados demostraron tres desigualdades básicas para la teoría de funciones cardinales. En sus versiones numerables ellos establecieron que los siguientes dos tipos de espacios no pueden tener cardinalidad mayor que $2^{\omega}$ : espacios de Hausdorff, primero numerables que satisfacen la ccc (condición de cadena numerable); espacios $T_{1}$ con pseudocarácter numerable (es decir, cuyos puntos son todos $G_{\delta}$ ) y tales que todos sus subespacios discretos son numerables. La versión numerable de la tercera desigualdad establece que un espacio de Hausdorff cuyos subespacios discretos son todos numerables no puede tener cardinalidad mayor que $2^{2^{\omega}}$.

En 1969 Arhangel'skiĩ resolvió el viejo problema de Alexandroff y Urysohn. En lenguaje de funciones cardinales él demostró que para todo espacio de Hausdorff $X$ se cumple que $|X| \leq 2^{L(X) \cdot x(X)}$. La versión numerable de esta desigualdad es la afirmación de que todo espacio de Hausdorff, Lindelöf, primero numerable tiene cardinalidad a lo sumo $2^{\omega}$.

$\mathrm{El}$ estudio de las funciones cardinales tiene varios aspectos: se analizan las relaciones entre las diferentes funciones cardinales; se utilizan las funciones cardinales para obtener cotas para la cardinalidad de los espacios, para el número de abiertos o abiertos regulares en un espacio; se estudia el comportamiento de las funciones cardinales en clases específicas de espacios topológicos, como los espacios compactos, metrizables, que satisfacen distintos axiomas de separación; se investiga el comportamiento de distintas funciones cardinales bajo operaciones como el producto o las uniones de cadenas crecientes de espacios.

Considerar uniones de cadenas crecientes de espacios es una idea muy natural. Con frecuencia, al estudiar un objeto, se trata de reducir ese objeto a objetos más simples que de alguna manera reflejen las propiedades del objeto más complejo. En nuestro caso, una de las maneras de hacer esto para estudiar un espacio topológico $X$, es descomponerlo en una unión de sus subespacios y analizar qué propiedades de los subespacios se "heredan" al espacio original. Un ejemplo de esto es el bien conocido resultado de que si un espacio es unión numerable de subespacios de Lindelöf, entonces el espacio es de Lindelöf. Claramente la misma conclusión no siempre es válida, por ejemplo la unión numerable o finita de espacios metrizables no necesariamente es un espacio metrizable.

Mikhail Tkachenko, en 1978, inició el estudio del comportamiento de las funciones cardinales bajo la operación de uniones de cadenas crecientes, estudio continuado más tarde por Hajnal y Juhász. Se planteó el siguiente problema general: supongamos que se tiene un espacio topológico $X$ que es la unión de una familia $\left\{X_{\alpha}: \alpha<\kappa\right\}$ de subespacios tales que $X_{\alpha} \subseteq X_{\beta}$ si $\alpha<\beta<\kappa$. $\mathrm{Si}$ se conocen los valores de alguna función cardinal en los sumandos, ¿qué se puede decir de $X$ ? 
El problema más inmediato en este contexto es tratar de expresar el valor de una función cardinal en $X$ en términos de los valores de esa función en los sumandos. Con respecto a esto, Tkachenko demostró que si la celularidad (celularidad hereditaria, densidad hereditaria, número de Lindelöf hereditario) de cada sumando $X_{\alpha}$ es estrictamente menor que algún cardinal infinito $\tau$, entonces la celularidad (celularidad hereditaria, densidad hereditaria, número de Lindelöf hereditario) de la unión no es mayor que $\tau$. También demostró afirmaciones análogas para la estrechez y el carácter cuando se supone que $X$ es un espacio compacto y Hausdorff.

A veces el valor de una función cardinal en $X$ depende no sólo de los valores de la función en los sumandos, sino también de la longitud de la cadena. Se sabe por ejemplo que si el pseudocarácter (peso de red) de cada sumando es $\leq \tau$ y la cadena tiene longitud $\kappa$, entonces el pseudocarácter (peso de red) de la unión no es mayor que $\tau \cdot \kappa$.

Otro problema consiste en establecer cotas para la cardinalidad del espacio en términos de los valores de alguna función cardinal en los sumandos. Se sabe, por ejemplo, que si $X$ es de Hausdorff y la densidad de cada sumando es a lo sumo $\tau$, entonces $|X| \leq 2^{2^{\tau}}$. Para espacios compactos y Hausdorff, Tkachenko probó que si el carácter de cada sumando es a lo sumo $\tau$, entonces $|X| \leq 2^{\tau}$.

$A$ veces se puede acotar el valor de una función cardinal en términos de los valores de otra función cardinal en los sumandos. Así tenemos, por ejemplo, que si $X$ es compacto y Hausdorff y la densidad de cada sumando es $\leq \tau$, entonces $w(X) \leq 2^{\tau}$.

Hay muchos resultados que acotan la cardinalidad de un espacio topológico en términos de funciones cardinales. Si $X$ es un espacio de Hausdorff, sabemos que $|X| \leq 2^{h L(X)},|X| \leq 2^{X(X) \cdot L(X)}$ y que $|X| \leq 2^{\psi(X) \cdot L(X) \cdot t(X)}$. Es natural preguntarse si estas desigualdades admiten generalizaciones en términos de cadenas crecientes de subespacios, es decir, si se puede obtener la cota imponiendo las restricciones de las funciones cardinales a los sumandos, no a todo el espacio. Se tienen algunos resultados a este respecto cuando $X$ un espacio Hausdorff que es la unión de una cadena creciente de subespacios $X_{\alpha}$. Hajnal y Juhász demostraron en 1981 que si $h L\left(X_{\alpha}\right) \leq \tau$ para cada $\alpha$, entonces $|X| \leq 2^{\tau}$ y si $\psi\left(X_{\alpha}\right) \cdot L\left(X_{\alpha}\right) \cdot t\left(X_{\alpha}\right) \leq \tau$ para cada $\alpha$, entonces $|X| \leq 2^{\tau}$ (ver [18]). Para la segunda desigualdad se tiene casi una generalización de este tipo, también de Hajnal y Juhász: se sabe que si $X$ es regular, $L(X) \leq \tau$ y $\chi\left(X_{\alpha}\right) \leq \tau$ para todo $\alpha$, entonces $|X| \leq 2^{\tau}$.

Los resultados se pueden mejorar si se imponen condiciones adicionales a los espacios. Por ejemplo, uno de los resultados de Tkachenko establece que si $X$ es un espacio de Hausdorff y el peso de red de cada sumando es estrictamente menor que $\tau$, entonces el peso del espacio es $\leq 2^{\tau}$. Pero si suponemos a $X$ no sólo Hausdorff sino compacto también, se puede mejorar la cota para el peso y concluir que $w(X) \leq \tau$. 
La presente investigación se enmarca en esta línea de pensamiento: mejorar los resultados conocidos imponiendo restricciones adecuadas a la unión o a los sumandos. Se obtuvieron resultados representativos de cada una de las líneas de investigación mencionadas arriba. El lema 3.4 y el teorema 3.5 expresan el valor de una función cardinal en términos de sus valores en los sumandos; el teorema 3.9 proporciona cotas para el espacio considerado en términos de los valores de una función cardinal en los sumandos; los teoremas 2.2, 2.4, 2.8, 2.10, 2.16, 3.7 y el corolario 2.17 acotan el valor de una función cardinal en términos de los valores de otras funciones cardinales en los sumandos y, finalmente, los teoremas 2.8 y 3.9 presentan generalizaciones de resultados conocidos en términos de uniones de cadenas de subespacios.

En el capítulo 2 consideramos espacios diádicos y grupos topológicos localmente compactos expresados como uniones de cadenas crecientes de sus subespacios y estudiamos el comportamiento de dos funciones cardinales, el pseudocarácter y la estrechez. Arhangel'skii y Ponomarev demostraron que la estrechez y el peso coinciden en la clase de los espacios diádicos, el teorema 2.8 es una generalización de este resultado. Los resultados principales del segundo capítulo son los siguientes:

- Si $X$ es un espacio diádico y el pseudocarácter (o la estrechez) de cada sumando es $\leq \tau$, entonces $w(X) \leq \tau$ (Teoremas 2.2 y 2.8).

- Si $G$ es un grupo topológico localmente compacto y el pseudocarácter (respectivamente, la estrechez) de cada sumando es $\leq \tau$, entonces $\chi(G) \leq \tau$ (Teoremas 2.4 y 2.10 ).

Mencionamos con anterioridad que no todas las propiedades topológicas son expresables por medio de cardinales, la metrizabilidad de un espacio es una de tales propiedades. Sin embargo, en el caso de los grupos topológicos, sí se puede dar una caracterización de la metrizabilidad por medio de funciones cardinales, gracias al teorema de Birkhoff- Kakutani que establece que un grupo topológico es metrizable si y sólo si es primero numerable. Para un grupo topológico $G$, ser metrizable equivale a tener carácter numerable. Tenemos, por lo tanto, como corolarios de los teoremas 2.4 y 2.10 , dos teoremas de metrizabilidad para grupos localmente compactos:

- Si $G$ es un grupo topológico localmente compacto que es la unión de una cadena creciente de sus subespacios y cada uno de ellos tiene pseudocarácter (o estrechez) numerable, entonces el grupo $G$ es metrizable (Corolarios 2.5 y 2.11).

En este mismo capítulo se presentan ejemplos que demuestran que los resultados obtenidos no son válidos para espacios compactos ni para grupos topológicos $\sigma$-compactos o numerablemente compactos.

En el capítulo 3 imponemos condiciones también a los sumandos de la cadena y analizamos el caso en el que un grupo topológico $G$ está expresado como unión de una cadena creciente de subgrupos. En este contexto podemos utilizar 
una función cardinal que es exclusiva para grupos topológicos, a saber, el índice de acotación de un grupo. Decimos que un grupo topológico $G$ es $\tau$-acotado si puede ser cubierto por a lo sumo $\tau$ traslaciones de cualquier vecindad de la identidad. El índice de acotación de $G$, $i b(G)$, es el mínimo cardinal $\tau$ tal que $G$ es $T$-acotado. Se prueba que si el índice de acotación de cada uno de los subgrupos de la cadena es estrictamente menor que $\lambda$, entonces el índice de acotación del grupo no es mayor que $\lambda$ (ver el teorema 3.5).

Una igualdad conocida para grupos topológicos es la siguiente: $w(G)=$ $i b(G) \cdot \chi(G)$ para todo grupo topológico $G$. Es natural preguntarse si esta igualdad admite una generalización en términos de cadenas crecientes, como la que se obtuvo para el teorema de Arhangel'skiī-Ponomarev. Lo mejor que se pudo obtener en este sentido es el teorema 3.7:

- Si $G$ es un grupo topológico expresable como unión de una cadena creciente de subespacios $\left\{G_{\alpha}: \alpha<\kappa\right\}, i b\left(G_{\alpha}\right) \cdot \chi\left(G_{\alpha}\right)<\lambda$ para toda $\alpha<\kappa$ y $c f(\kappa) \neq \lambda^{+}$, entonces $w(G) \leq 2^{\lambda}$.

El ejemplo 3.8 muestra que el resultado no se puede mejorar, es decir, no se puede reducir la cota ni se puede quitar la restricción acerca de la cofinalidad de la longitud de la cadena.

La parte final del capítulo 3 se dedica al problema de encontrar cotas para la cardinalidad de los grupos. Se sabe que para todo grupo topológico $G$ se tiene que $|G| \leq 2^{i b(G) \cdot \psi(G)}$. Con el teorema 3.9 damos una generalización de este resultado en términos de cadenas crecientes para grupos numerablemente compactos y con el ejemplo 3.10 mostramos que esta generalización no es válida en general, ni siquiera para grupos pseudocompactos.

Muchos son los resultados de grupos topológicos, espacios compactos diádicos y teoría de funciones cardinales que se utilizan a lo largo de la tesis. El capítulo 1 está dedicado a exponer, de la manera más detallada posible, todo este material. Se definen todas las funciones cardinales con las que se trabaja, se demuestran algunas de sus propiedades más importantes, se definen los espacios diádicos y los grupos topológicos y se enuncian los teoremas relativos al comportamiento de las funciones cardinales en estas clases de espacios. 


\section{CAPÍTULO 1}

\section{Preliminares}

Bertrand Russell dijo que todo libro debe ser inteligible o correcto, pero que es imposible que sea ambas cosas a la vez. A riesgo de pecar de arrogante, pretendo lograr ambas cualidades para este trabajo, y este capítulo es mi intento de conseguir la primera.

El objetivo de este capítulo es dar una referencia completa de los resultados utilizados en los siguientes capítulos. La primera sección está destinada a fijar la notación que se seguirá, así como a dar algunas definiciones de conceptos básicos, tanto de teoría de conjuntos como de topología. En la segunda sección se definen las funciones cardinales con las que se trabajará y se presentan los resultados conocidos más importantes. Las siguientes dos secciones tratan, respectivamente, sobre cubos generalizados de Cantor y grupos topológicos. Finalmente, en la quinta sección se incluyen resultados acerca del comportamiento de las funciones cardinales bajo la operación de tomar uniones de cadenas crecientes de espacios.

La elección del material ha estado determinada de manera pragmática: se ha incluido únicamente lo que se va a necesitar posteriormente. La mayoría de los resultados están demostrados. Los teoremas que requerirían una desviación demasiado larga para su demostración aparecen sólo enunciados, pero con una referencia a alguna fuente que se puede consultar para su demostración.

\section{Notación y definiciones}

A lo largo del trabajo adoptamos la notación usual en teoría de conjuntos: $\kappa, \lambda, \mu, \nu$ y $\tau$ son cardinales; $\alpha, \beta, \gamma$ y $\delta$ son ordinales; $\omega$ es el mínimo ordinal infinito y $\aleph_{0}$ denota al mínimo cardinal infinito, aunque a veces se usa $\omega$ para referirse al cardinal; $\omega_{1}$ es el mínimo ordinal y cardinal no numerable; $c$ denota a la cardinalidad del continuo, es decir, $c=2^{\aleph_{0}} ; \kappa^{+}$es el mínimo cardinal mayor que $\kappa$. Cada ordinal es el conjunto de los ordinales menores que él, por lo que $\alpha<\beta$ y $\alpha \in \beta$ son lo mismo.

Un ordinal $\alpha$ es un ordinal sucesor si $\alpha=\beta+1$ para algún ordinal $\beta$. Cuando un ordinal no es un ordinal sucesor, se dice que es un ordinal límite. Si $\alpha$ es un ordinal límite, entonces, para todo ordinal $\beta$ se cumple que $\beta \in \alpha$ implica $\beta+1 \in \alpha$. Todo cardinal infinito es un ordinal límite.

Un cardinal $\kappa$ es un cardinal sucesor si $\kappa=\lambda^{+}$para algún cardinal $\lambda$. Por ejemplo, $\omega_{1}$ es un cardinal sucesor, pues $\omega_{1}=\omega^{+}$. Si un cardinal no es un 
cardinal sucesor entonces es un cardinal límite. Así, $\kappa$ es un cardinal límite si $\lambda<\kappa$ implica que $\lambda^{+}<\kappa$.

La cofinalidad de $\kappa$, denotada $\operatorname{cf}(\kappa)$, es el mínimo cardinal $\lambda$ tal que $\kappa$ tiene un subconjunto cofinal de cardinalidad $\lambda$. Por ejemplo, $\operatorname{cf}(\omega)=\omega$ y $\operatorname{cf}\left(\kappa^{+}\right)=\kappa^{+}$ para todo cardinal $\kappa \geq \omega$. Un cardinal $\kappa$ es regular $\operatorname{si} \kappa \geq \omega$ y $\operatorname{cf}(\kappa)=\kappa$. Una propiedad importante de cualquier cardinal regular $\kappa$ es que si $A \subseteq \kappa$ y $A$ tiene cardinalidad estrictamente menor que $\kappa$, entonces $\sup (A)<\kappa$. Se tiene que $\operatorname{cf}(\kappa)$ es in cardinal regular para todo $\kappa \geq \omega$.

También usaremos la notación y las convenciones topológicas usuales: $X$ siempre denota a un espacio topológico no vacío; los espacios compactos son de Hausdorff; si $\left\{X_{i}: i \in I\right\}$ es una familia de espacios topológicos, el producto de la familia con la topología usual de producto se escribe $\Pi\left\{X_{i}: i \in I\right\}$ o $\prod_{i \in I} X_{i}$ y las proyecciones naturales se denotan indistintamente por $p_{i}$ o por $\pi_{i}$. Si $X$ es un espacio topológico y $A \subseteq X, \operatorname{Int}(A)$ y $\bar{A}$ denotan al interior y a la cerradura de $A$, respectivamente. Al conjunto de todos los abiertos de $X$ lo denotamos $\tau(X)$ y, para cada $p \in X, \tau(p, X)$ es el conjunto de los abiertos de $X$ que contienen al punto $p$.

En lo que sigue $D$ se usará para denotar al espacio $\{0,1\}$ con la topología discreta. El cubo generalizado de Cantor de peso $\mu$, donde $\mu$ es cualquier cardinal infinito, se obtiene tomando el producto de $\mu$ copias de $D$ y se denota $D^{\mu}$. Tiene cardinalidad $2^{\mu}$, tiene una base de cardinalidad $\mu$ y no tiene ninguna base de cardinalidad $<\mu$.

Por último, dado un cardinal infinito $\mu, A(\mu)$ denota la compactificación en un punto (compactificación de Alexandroff) de un espacio discreto de cardinalidad $\mu$.

\section{Funciones cardinales}

Esta sección no pretende ser un curso introductorio a las funciones cardinales, muchas funciones cardinales importantes ni siquiera se mencionan. La idea aquí es definir todas las funciones cardinales con las que se trabajará y presentar los resultados que serán utilizados en los capítulos posteriores. Para un tratamiento completo de los hechos fundamentales de funciones cardinales se puede consultar [18].

Una función cardinal es una función $\phi$ definida en una clase de espacios topológicos y que toma valores en la clase de todos los cardinales infinitos de tal modo que, si $X$ y $Y$ son dos espacios homeomorfos, entonces $\phi(X)=\phi(Y)$. La petición de que las funciones cardinales tomen siempre valores infinitos simplifica los enunciados de los teoremas y pone énfasis en la aritmética cardinal transfinita. En vista de esta convención, la función cardinal más evidente, que es precisamente la cardinalidad del espacio $X$, se define como el número de puntos del espacio $+\omega$. La cardinalidad de $X$ se denota $|X|$. 
Probablemente la función cardinal más útil es el peso de $X$, denotado $w(X)$, y que se define como

$$
w(X)=\min \{|\mathcal{B}|: \mathcal{B} \text { es una base de } X\}+w .
$$

Ninguna de estas dos funciones domina a la otra, es decir, ni $w(X) \leq|X|$ ni $|X| \leq w(X)$ son desigualdades válidas para todo espacio $X$. Sin embargo, si suponemos que $X$ es $T_{0}$, no difieren en más de un exponente. Es claro que $w(X) \leq 2^{|X|}$ para todo $X$, si además $X$ es $T_{0}$ y $\mathcal{B}$ es una base de $X$ con $|\mathcal{B}|=w(X)$, entonces la función $\Phi: X \rightarrow P(\mathcal{B})$ definida como $\Phi(p)=\{B \in$ $B: p \in B\}$ es inyectiva y en consecuencia $|X| \leq 2^{w(X)}$. De aquí se desprende el siguiente teorema.

Teorema 1.1. Para todo espacio topológico $X$ se tiene que $w(X) \leq 2^{|X|}$. Si además $X$ es $T_{0}$, entonces $|X| \leq 2^{w(X)}$.

La siguiente función cardinal es una generalización natural del peso de un espacio. Una red para un espacio topológico $X$ es una familia $\mathcal{R}$ de subconjuntos de $X$ tal que, para todo punto $p \in X$ y todo abierto $U$ de $X$ tal que $p \in U$, existe $R \in \mathcal{R}$ con $p \in R \subseteq U$. Definimos el peso de red de un espacio topológico $X$, denotado por $n w(X)$, como sigue:

$$
n w(X)=\min \{|\mathcal{R}|: \mathcal{R} \text { es una red para } X\}+\omega .
$$

Cualquier base de $X$ es una red para $X$, una red que consiste de conjuntos abiertos. El conjunto de todos los subconjuntos unitarios de $X$ es otro ejemplo de una red para $X$. Estos dos ejemplos muestran que $n w(X) \leq w(X)$ y $n w(X) \leq|X|$ para todo espacio $X$.

Otra función cardinal importante es la densidad de $X$. La densidad de un espacio $X$ es la mínima cardinalidad de un subconjunto denso en $X$, es decir:

$$
d(X)=\min \{|S|: S \subseteq X, \bar{S}=X\}+\omega .
$$

Un espacio $X$ es separable si y sólo si $d(X)=\omega$. Claramente si $\mathcal{R}$ es una red para $X$ y escogemos un punto de cada miembro de $\mathcal{R}$, el conjunto resultante es denso en $X$, por lo que tenemos las siguientes desigualdades para todo espacio $X$ :

TEOREMA 1.2. En todo espacio $X$ se cumplen las siguientes desigualdades:

(a) $d(X) \leq n w(X) \leq w(X)$;

(b) $d(X) \leq n w(X) \leq|X|$.

El primer inciso del teorema anterior es una generalización en el lenguaje de funciones cardinales del conocido resultado de que todo espacio segundo numerable es separable.

El siguiente resultado de la teoría de funciones cardinales se puede ver como un "inverso" del teorema anterior.

TEOREMA 1.3. Si $X$ es un espacio regular, entonces $w(X) \leq 2^{d(X)}$. 
Demostración. Sea $S \subseteq X$ un conjunto denso en $X$ tal que $|S|=d(X)$. Sea $\mathcal{B}=\{\operatorname{Int}(\overline{V \cap S}): V \in \tau(X)\}$. Nótese que $|\mathcal{B}| \leq|P(S)|=2^{|S|}$. Afirmamos que $\mathcal{B}$ es una base de $X$. Para ver esto, consideremos un abierto no vacío cualquiera $U \subseteq X$ y tomemos $x \in U$. Como $X$ es regular, existe un conjunto abierto $V \subseteq X$ tal que $x \in V \subseteq \bar{V} \subseteq U$. Pero $S$ es denso en $X$ y $V$ es abierto, por lo que $\bar{V}=\overline{V \cap S}$. Por lo tanto $x \in \operatorname{Int}(\overline{V \cap S}) \subseteq U$. Esto prueba que $\mathcal{B}$ es una base para la topología de $X$ y en consecuencia $w(X) \leq|\mathcal{B}| \leq 2^{|S|}=$ $2^{d(X)}$.

La siguiente función cardinal, a diferencia del peso, peso de red o densidad, no está definida como un mínimo, sino como un supremo de cardinales. La pregunta acerca de cuándo se alcanza el supremo, es decir, cuándo el supremo es de hecho un máximo, se conoce como el problema "sup=max". No es un problema relevante para nosotros.

Una colección de subconjuntos abiertos no vacíos de $X$, ajenos dos a dos es una familia celular. La celularidad del espacio $X$ se define de la siguiente manera:

$$
c(X)=\sup \{|\mathcal{A}|: \mathcal{A} \text { es una familia celular en } X\}+\omega \text {. }
$$

Un espacio $X$ satisface la ccc (condición de cadena numerable) si $c(X)=\omega$. Si $\mathcal{A}$ es una familia celular en $X$ y $D$ es un subconjunto denso de $X$, entonces para cada $A \in \mathcal{A}, A \cap D \neq \emptyset$. Como la familia $\mathcal{A}$ es ajena, concluimos que $|\mathcal{A}| \leq|D|$ y obtenemos que $c(X) \leq d(X)$ para todo espacio $X$.

Las funciones cardinales hasta ahora definidas son funciones globales, es decir, sus definiciones se basan en propiedades topológicas que proporcionan información global acerca del espacio. Ciertas funciones cardinales tienen un carácter local, es decir, están basadas en propiedades locales del espacio. Estas funciones se evalúan en cada punto del espacio y después se define la función en el espacio como el supremo de los valores que toma en los puntos del espacio.

Sea $X$ un espacio topológico y $A$ un subconjunto arbitrario de $X$. Una base local para $A$ es una familia $B$ de subconjuntos abiertos de $X$ que contienen a $A$ tal que para todo abierto $U \subseteq X$, si $A \subseteq U$ entonces existe $B \in \mathcal{B}$ tal que $A \subseteq B \subseteq U$. El carácter del conjunto $A \subseteq X$ se define como la mínima cardinalidad de una base local para $A$ y se denota $\chi(A, X)$. Si $p \in X$, escribimos $\chi(p, X)$ en vez de $\chi(\{p\}, X)$. El carácter del espacio $X$ se define del siguiente modo:

$$
\chi(X)=\sup \{\chi(p, X): p \in X\}+\omega .
$$

Un espacio $X$ es primero numerable si y sólo si $\chi(X)=\omega$.

Una pseudobase local para $A$ en un espacio $T_{1} X$ es una familia $B$ de subconjuntos abiertos de $X$ que contienen a $A$ tal que $A=\cap B$. El pseudocarácter del conjunto $A \subseteq X$ se define como la mínima cardinalidad de una pseudobase local para $A$ y se denota $\psi(A, X)$. El pseudocarácter del espacio $X$ se define 
como

$$
\psi(X)=\sup \{\psi(p, X): p \in X\}+\omega .
$$

Obsérvese que la definición de pseudocarácter sólo tiene sentido para espacios $T_{1}$. Si B es una base para la topología de $X$ y $p$ es un punto de $X$, entonces claramente $\{B \in \mathcal{B}: p \in B\}$ es una base local para $p$. Además, si el espacio es $T_{1}$, toda base local es una pseudobase local. Por lo tanto, en la clase de los espacios $T_{1}$ se tiene que $\psi(X) \leq \chi(X) \leq w(X)$.

Definimos ahora la última de las funciones cardinales con las que trabajaremos. La estrechez de $p$ en $X$, en símbolos $t(p, X)$ es

$$
\begin{gathered}
t(p, X)=\min \{\kappa: \text { para todo } Y \subseteq X \text { con } p \in \bar{Y}, \text { existe } \\
A \subseteq Y \text { con }|A| \leq \kappa \text { y } p \in \bar{A}\} .
\end{gathered}
$$

La estrechez del espacio $X$ está dada por:

$$
t(X)=\sup \{t(p, X): p \in X\}+\omega .
$$

Es fácil ver que $t(X) \leq \chi(X)$ para todo $X$. Consideremos un subconjunto $Y \subseteq X$, un punto $p \in \bar{Y}$ y una base local $\mathcal{B}$ para $p$ en $X$, tal que $|\mathcal{B}| \leq \chi(p, X)$. Es claro que para todo $B \in \tau(p, X), B \cap Y \neq \emptyset$. Si elegimos un punto $x_{B} \in B \cap Y$ para cada $B \in \mathcal{B}$ y hacemos $A=\left\{x_{B}: B \in \mathcal{B}\right\}$, es claro que $A \subseteq Y$, $|A| \leq|\mathcal{B}| \leq \chi(p, X)$ y $p \in \bar{A}$. Por lo tanto $t(p, X) \leq \chi(p, X)$ para todo $p \in X$ $y$, en consecuencia, $t(X) \leq \chi(X)$.

De las funciones que hemos definido, el peso, el peso de red, el pseudocarácter, el carácter y la estrechez son funciones monótonas. Esto quiere decir que si $X$ es un espacio topológico y $Y \subseteq X$, entonces, $w(Y) \leq w(X)$, $n w(Y) \leq n w(X), \psi(Y) \leq \psi(X), \chi(Y) \leq \chi(X)$ y $t(Y) \leq t(X)$. Esto es consecuencia inmediata de las definiciones.

No todas las funciones cardinales son monótonas, la densidad y la celularidad son ejemplos de funciones que no lo son. Para ver esto basta recordar que el plano de Niemytzki es separable, tiene celularidad numerable y contiene un subespacio discreto de cardinalidad c, lo que implica que ese subespacio no es separable ni satisface la ccc.

A continuación vamos a demostrar dos resultados muy sencillos acerca del comportamiento de algunas funciones cardinales bajo mapeos continuos.

Proposición 1.4. Si $f: X \longrightarrow Y$ es un mapeo continuo e inyectivo entre espacios $T_{1}$, entonces $\psi(X) \leq \psi(Y)$.

Demostración. Vamos a probar que $\psi(p, X) \leq \psi(Y)$ para todo punto $p \in X$. Tomemos un punto $p \in X$ y consideremos $f(p) \in Y$. Sea $\mathcal{A}$ una familia de abiertos de $Y$ tal que $|\mathcal{A}| \leq \psi(Y)$ y $\{f(p)\}=\bigcap \mathcal{A}$. Entonces $\mathcal{C}=\left\{f^{-1}(A)\right.$ : $A \in \mathcal{A}\}$ es una familia de abiertos en $X$ y $|\mathcal{C}| \leq \psi(Y)$. Para ver que $\cap \mathcal{C}=\{p\}$, basta notar que si $q \in \cap \mathcal{C}$, entonces $f(q) \in A$ para todo conjunto $A \in \mathcal{A}$ y por lo tanto $f(q)=f(p)$. Dado que $f$ es inyectiva, tenemos que $p=q$. 
Proposición 1.5. Si $f: X \longrightarrow Y$ es un mapeo continuo, abierto $y$ suprayectivo, entonces $t(Y) \leq t(X)$.

Demostración. Supongamos que $t(X)=\kappa$. Consideremos cualquier subconjunto $A \subseteq Y$ y supongamos que $y \in \bar{A}$. Sea $x \in X$ tal que $f(x)=y$. Entonces $x \in f^{-1}(\bar{A})$ y, en vista de que $f$ es abierta, $x \in \overline{f^{-1}(A)}$. Como $t(X)=\kappa$, existe $B \subseteq f^{-1}(A)$ tal que $|B| \leq \kappa$ y $x \in \bar{B}$. Claramente $f(B) \subseteq A$, $|f(B)| \leq|B| \leq \kappa$ y directamente se verifica que $y \in \overline{f(B)}$. Por lo tanto $t(y, Y) \leq \kappa$ para todo punto $y \in Y$.

Cuando se imponen restricciones al espacio, el comportamiento de las funciones cardinales mejora. Por ejemplo, el teorema 1.3 no es válido para espacios de Hausdorff. Para estos espacios, el mejor resultado que se puede obtener para acotar el peso en términos de la densidad es que $w(X) \leq 2^{2^{d(X)}}$.

Para la clase de los espacios compactos, Alexandroff demostró que el carácter y el pseudocarácter coinciden y que el peso no puede ser mayor que la cardinalidad del espacio. Arhangel'skii probó que se puede dar una caracterización de la estrechez de un espacio en términos de sucesiones libres. Por definición, una sucesión de puntos de un espacio $X,\left\{x_{\alpha}: \alpha<\kappa\right\}$ es una sucesión libre de longitud $\kappa$ si para toda $\beta<\kappa, \overline{\left\{x_{\alpha}: \alpha<\beta\right\}} \cap \overline{\left\{x_{\alpha}: \beta \leq \alpha<\kappa\right\}}=\emptyset$. Nótese que toda sucesión libre es un subespacio discreto de $X$. Se define entonces $F(X)$ como el supremo de todos los cardinales $\kappa$ tales que $X$ tiene una sucesión libre de longitud $\kappa$. El resultado de Arhangel'skii es el siguiente:

TeOrema 1.6. Si $X$ es un espacio compacto, entonces $t(X)=F(X)$.

En las siguientes dos secciones vamos a analizar el comportamiento de estas funciones cardinales en dos clases de espacios topológicos: los cubos generalizados de Cantor y los grupos topológicos.

\section{Cubos generalizados de Cantor}

Sea $D$ el espacio $\{0,1\}$ con la topología discreta. El cubo generalizado de Cantor de peso $\mu$, donde $\mu$ es cualquier cardinal infinito, se obtiene tomando el producto de $\mu$ copias de $D$ con la topología usual para el producto y se denota $D^{\mu}$. Para cada $\alpha<\mu, \pi_{\alpha}: D^{\mu} \longrightarrow D$ es la proyección natural sobre el $\alpha$-ésimo factor. Las funciones cardinales se comportan muy bien en la clase de los cubos generalizados de Cantor. En esta sección vamos a demostrar que $\psi\left(D^{\mu}\right)=\chi\left(D^{\mu}\right)=w\left(D^{\mu}\right)=t\left(D^{\mu}\right)=\mu$.

Como el conjunto de todos los subconjuntos finitos de $\mu$ tiene cardinalidad $\mu$ para cualquier $\mu$ infinito, la base canónica para $D^{\mu}$ tiene cardinalidad $\mu$. Esto significa que $w\left(D^{\mu}\right) \leq \mu$. Por otro lado, para cada $\alpha<\mu$ podemos definir un punto $p_{\alpha} \in D^{\mu}$ estipulando que $p_{\alpha}(\alpha)=1$ y $p_{\alpha}(\beta)=0$ para todo $\beta<\mu$ con $\beta \neq \alpha$. Entonces el conjunto $S=\left\{p_{\alpha}: \alpha<\mu\right\}$ es un subespacio discreto de $D^{\mu}$ de cardinalidad $\mu$ y, en consecuencia, $w\left(D^{\mu}\right) \geq \mu$. Hemos establecido 
que el nombre 'cubo generalizado de Cantor de peso $\mu$ ' es apropiado, es decir, acabamos de demostrar el siguiente teorema.

Teorema 1.7. Para todo cardinal infinito $\mu, w\left(D^{\mu}\right)=\mu$.

Consideremos un punto arbitrario $p \in D^{\mu}$ y tomemos una familia $\mathcal{A} \subseteq$ $\tau\left(p, D^{\mu}\right)$ de abiertos básicos canónicos tal que $|\mathcal{A}|=\kappa<\mu$. Para cada $U \in \mathcal{A}$, hagamos $\operatorname{coord}(U)=\left\{\alpha<\mu: \pi_{\alpha}(U) \neq D\right\}$ e $I=U\{\operatorname{coord}(U): U \in \mathcal{A}\}$. Entonces $|I| \leq \kappa<\mu$ y por lo tanto $\mu \backslash I \neq \emptyset$. Podemos entonces definir a $q \in$ $D^{\mu}$ de tal forma que $q(\alpha)=p(\alpha)$ si $\alpha \in I$ y $q(\alpha) \neq p(\alpha)$ si $\alpha \in \mu \backslash I$. Claramente $p \neq q$ y $q \in \cap \mathcal{A}$. Por lo tanto $\cap \mathcal{A} \neq\{p\}$. Como cada conjunto abierto $A \in \tau\left(p, D^{\mu}\right)$ contiene un abierto básico canónico, lo demostrado anteriormente implica que $\psi\left(p, D^{\mu}\right) \geq \mu$. Pero $\psi\left(D^{\mu}\right) \leq \chi\left(D^{\mu}\right) \leq w\left(D^{\mu}\right)=\mu$, por lo tanto tenemos el siguiente teorema.

TEOREMA 1.8. Para todo cardinal infinito $\mu, \psi\left(D^{\mu}\right)=\chi\left(D^{\mu}\right)=w\left(D^{\mu}\right)=$ $\mu$.

Para evaluar la celularidad de $D^{\mu}$ se necesita el famoso teorema de Hewitt, Marczewski y Pondiczery acerca de la densidad de productos de espacios, mencionado en la introducción. Este teorema afirma que si $X=\prod_{a \in A} X_{a}$, $d\left(X_{a}\right) \leq \kappa$ para cada $a \in A$ y $|A| \leq 2^{\kappa}$, entonces $d(X) \leq \kappa$. Se puede consultar la demostración de este teorema en el segundo capítulo de [11].

TEOREMA 1.9. Para todo cardinal infinito $\mu, c\left(D^{\mu}\right)=\aleph_{0}$.

Demostración. Sea $\mathcal{A}$ una familia celular de abiertos de $D^{\mu}$ tal que $|\mathcal{A}|=\omega_{1}$. Cada elemento de $\mathcal{A}$ contiene un abierto básico canónico, por lo que podemos suponer, sin perder generalidad, que los elementos de $\mathcal{A}$ son todos básicos canónicos. De la misma manera que en el teorema 1.7, para cada $U \in \mathcal{A}$, hagamos $\operatorname{coord}(U)=\left\{\alpha<\mu: \pi_{\alpha}(U) \neq D\right\}$ e $I=U\{\operatorname{coord}(U): U \in \mathcal{A}\}$. Entonces $|I| \leq \omega_{1} \leq 2^{\omega}$. Ahora tomemos el producto $X=\prod_{\alpha \in I} D$, que es separable en virtud del teorema de Hewitt, Marczewski y Pondiczery. Sea $\pi_{I}: D^{\mu} \longrightarrow X$ la proyección natural. Por la elección de $I$, si $U \in \mathcal{A}$ y $\alpha \in \mu \backslash I$, entonces $\pi_{\alpha}(U)=D$. Esto implica que la familia $\mathcal{A}^{*}=\left\{\pi_{I}(U): U \in \mathcal{A}\right\}$ es una familia celular en $X$ de cardinalidad igual a la cardinalidad de $\mathcal{A}$. Como $c(X) \leq d(X)$ (ver la sección 2), concluimos que $|\mathcal{A}| \leq \omega$. Esta contradicción demuestra que en $D^{\mu}$ no hay familias celulares de cardinalidad $\omega_{1}$ y por lo tanto $c\left(D^{\mu}\right)=\aleph_{0}$.

La otra función cardinal que nos interesa es la estrechez. La estrechez es siempre menor o igual al peso, como se vio en la sección 2, pero en el caso de los cubos generalizados de Cantor se tiene la igualdad de las dos funciones.

Teorema 1.10. Para todo cardinal infinito $\mu, t\left(D^{\mu}\right)=\mu$. 
Demostración. Basta probar que $t\left(D^{\mu}\right) \geq \mu$. Sea $\mathcal{F}=\left\{I \subseteq \mu:|I|<\aleph_{0}\right\}$. Entonces $|\mathcal{F}|=\mu$ y por lo tanto podemos escribir a $\mathcal{F}$ de la siguiente manera:

$$
\mathcal{F}=\left\{I_{\alpha}: \alpha<\mu\right\} .
$$

Tomemos un punto arbitrario $p \in D^{\mu} \mathrm{y}$, para cada $\alpha<\mu$, definamos un punto $p_{\alpha} \in D^{\mu}$ haciendo

$$
\begin{aligned}
& p_{\alpha}(\beta)=p(\beta) \text { si } \beta \in I_{\alpha}, \\
& p_{\alpha}(\beta) \neq p(\beta) \text { si } \beta \notin I_{\alpha} .
\end{aligned}
$$

Sea $M=\left\{p_{\alpha}: \alpha<\mu\right\}$. Claramente $M$ tiene cardinalidad $\mu$. Para ver que $p \in \bar{M}$, definamos $U_{\alpha}=\bigcap\left\{\pi_{\beta}^{-1}(\{p(\beta)\}): \beta \in I_{\alpha}\right\}$ para cada $\alpha<\mu$. Sólo resta notar que cada abierto básico canónico $U \in \tau\left(p, D^{\mu}\right)$ es igual a algún $U_{\alpha}$ y que para cada $\alpha<\mu, p_{\alpha} \in U_{\alpha} \cap M$. Ahora consideremos cualquier subconjunto $N \subseteq M$ con $|N|<\mu$. Entonces $\left|\bigcup\left\{I_{\alpha}: p_{\alpha} \in N\right\}\right| \leq|N|<\mu$ y por lo tanto podemos elegir $\beta \in \mu \backslash \bigcup\left\{I_{\alpha}: p_{\alpha} \in N\right\}$. Por definición, para cada $p_{\alpha} \in N$, $p_{\alpha}(\beta) \neq p(\beta)$. Esto quiere decir que $N \cap \pi_{\beta}^{-1}(\{p(\beta)\})=\emptyset$ y por lo tanto $p \notin \bar{N}$. Concluimos que $t\left(p, D^{\mu}\right) \geq \mu$.

De manera análoga a la demostración del teorema 1.7, dado cualquier punto $p \in D^{\mu}$ se puede definir un subespacio discreto de $D^{\mu}, M=\left\{p_{\alpha}: \alpha<\mu\right\}$, haciendo, para cada $\alpha<\mu, p_{\alpha}(\alpha) \neq p(\alpha)$ y $p_{\alpha}(\beta)=p(\beta)$ para $\beta \neq \alpha$. La cardinalidad de $M$ es $\mu$ y $p$ es un punto de acumulación de $M$. Recordemos que $A(\mu)$ es la compactificación de Alexandroff del espacio discreto de cardinalidad $\mu$. Entonces tenemos el siguiente teorema.

Teorema 1.11. Si $p \in D^{\mu}$, entonces existe un subespacio $M \subseteq D^{\mu}$ de cardinalidad $\mu$ tal que $M$ es discreto y $M \cup\{p\}$ es homeomorfo a $A(\mu)$.

Un espacio compacto $X$ se llama espacio diádico si es imagen continua del cubo generalizado de Cantor $D^{\mu}$ para algún $\mu \geq \aleph_{0}$. Este concepto fue introducido por Alexandroff ([1]) en 1936. Los espacios diádicos tienen todas las propiedades que hemos demostrado para los cubos generalizados de Cantor: la celularidad de todo espacio diádico es numerable; en un espacio diádico coinciden el peso, la estrechez y el carácter (éste es el teorema de Arhangel'skii-Ponomarev en [2]) y en 1965 Efimov demostró el teorema 1.11 para cualquier espacio diádico $X$ y cualquier punto $p \in X$ tal que $\chi(p, X)=\mu$.

El teorema crucial para la demostración de los teoremas 2.2 y 2.8 es el teorema de Efimov, Gerlitz y Hagler (ver [10], [13] o [15]) que afirma que todo espacio diádico de peso regular contiene una copia topológica del cubo generalizado de Cantor del mismo peso. Una demostración detallada del teorema también se puede consultar en [22].

TEOREma 1.12. Si $X$ es un espacio compacto diádico, $x \in X, \chi(x, X) \geq \mu$ $y \mu$ es un cardinal regular, entonces existe un subespacio $Y \subseteq X$ tal que $Y \approx D^{\mu}$ $y x \in Y$. 


\section{Grupos topológicos}

Un grupo $(G, \cdot)$ con una topología $\tau$ es un grupo topológico si tanto el mapeo de multiplicación $(x, y) \mapsto x \cdot y$ como el mapeo del inverso $x \mapsto x^{-1}$ son continuos con respecto a $\tau$. Esto último equivale a decir que el mapeo $\phi$ de $G^{2}$ en $G$ definido por $\phi(x, y)=x \cdot y^{-1}$ es continuo.

En un grupo topológico $G$, la operación $\psi$ definida como $\psi(x)=x^{-1}$ es un homeomorfismo, pues por definición es continua y $\psi \circ \psi=\mathrm{id}_{G}$.

Para cualquier subconjunto $V \subseteq G$, es usual escribir $V^{-1}$ en lugar de $\psi(V)$. Un subconjunto $V \subseteq G$ es simétrico si $V^{-1}=V$. Nótese que el conjunto $U \cap U^{-1}$ es simétrico para cada $U \subseteq G$. Como la operación del inverso en $G$ es continua en $G, U^{-1}$ es abierto para cada conjunto abierto $U \subseteq G$, concluimos que los conjuntos abiertos simétricos constituyen una base local de la identidad en $G$.

Si $A, B \subseteq G$, el producto de $A$ y $B$ en $G$ se define como sigue:

$$
A \cdot B=\{a \cdot b: a \in A, b \in B\} .
$$

Cuando $A=B$, escribimos $A^{2}$ en vez de $A \cdot A$. Como la multiplicación en $G$ es continua, para cada vecindad $U$ de la identidad de $G$ existe una vecindad de la identidad $V$ tal que $V^{2} \subseteq U$; además se puede suponer que la vecindad $V$ es simétrica.

Dado un grupo topológico $G$, para cada elemento $g \in G$ definimos la traslación izquierda $t_{g}: G \longrightarrow G$ por $t_{g}(x)=g \cdot x$ para todo $x \in G$. Por la continuidad de la multiplicación en $G$, el mapeo $t_{g}$ es continuo para cada $g \in G$. Note además que $t_{g^{-1}} \circ t_{g}=i d_{G}$ y que $t_{g} \circ t_{g^{-1}}=i d_{G}$. Como $t_{g}$ es una biyección de $G$, podemos concluir que la traslación $t_{g}$ es un homeomorfismo de $G$. Una consecuencia inmediata de este hecho es la homogeneidad de los grupos topológicos.

PRoposición 1.13. Todo grupo topológico es homogéneo, es decir, para cualesquiera $x, y \in G$ existe un homeomorfismo $f: G \longrightarrow G$ tal que $f(x)=y$.

Demostración. Considérese la traslación $t_{g}$ de $G$ para $g=y \cdot x^{-1}$.

Si $U \subseteq G$ y $x \in G$, el conjunto $t_{x}(U)$ generalmente se denota por $x U$. Obsérvese que si $U$ es abierto, entonces también lo es $x U$ para todo punto $x \in$ $G$. Los conjuntos $U x$ se definen de manera análoga por medio de traslaciones derechas.

Claramente si consideramos la topología discreta o la topología antidiscreta en cualquier grupo, éste se convierte en un grupo topológico. Pero para que la teoría sea más rica necesitamos imponer ciertas condiciones de separación. El teorema que sigue muestra que es suficiente con pedir que la identidad del grupo sea un conjunto cerrado.

TEOREMA 1.14. Sea $G$ un grupo topológico con identidad e. Si $\{e\}$ es un conjunto cerrado en $G$, entonces $G$ es un espacio topológico regular. 
Demostración. Como $G$ es homogéneo, todos los puntos de $G$ son cerrados y por lo tanto $G$ es un espacio $T_{1}$. Para verificar la regularidad de $G$, basta verificar la regularidad de $G$ en la identidad $e$. Para esto consideremos una vecindad abierta $U$ de $e$. Por la continuidad de la multiplicación, existe una vecindad abierta $V$ de $e$ tal que $V^{2} \subseteq U$. Entonces $W=V \cap V^{-1}$ es una vecindad abierta y simétrica de la identidad. Afirmamos que $\bar{W} \subseteq U$. En efecto, sea $x \in \bar{W}$ arbitrario. Entonces $x W$ es abierto, por lo que $x W \cap W \neq \emptyset$. Escojamos $a, b \in W$ tales que $x \cdot a=b$. Entonces $x=b \cdot a^{-1} \in W \cdot W^{-1}=$ $W^{2} \subseteq V^{2} \subseteq U$.

Note que el grupo $G$ en el teorema 1.14 es Hausdorff. Por lo tanto, para cualquier grupo topológico las propiedades de ser regular, Hausdorff o $T_{1}$ coinciden. Nosotros vamos a suponer siempre que los grupos son Hausdorff.

Un subgrupo de un grupo topológico $G$ es un subgrupo (algebraico) de $G$ con la topología de subespacio. Es inmediato que todo subgrupo de un grupo topológico es a su vez un grupo topológico. Si $H$ es un subgrupo cerrado y normal (algebraicamente) de $G$, el grupo cociente $G / H$ consiste de las clases laterales izquierdas $x H, x \in G$. Sea $\pi$ el homomorfismo natural de $G$ en $G / H$, $\pi(x)=x H$ para cada $x \in G$. Entonces podemos introducir una topología en $G / H$ estipulando que el conjunto $V \subseteq G / H$ sea abierto en $G / H$ si y sólo si $\pi^{-1}(V)$ es abierto en $G$. Se verifica fácilmente que el grupo $G / H$ con esta topología es un grupo topológico. Note que la identidad de $G / H, \pi(e)$ es cerrada en $G / H$ porque $\pi^{-1}(\pi(e))=H$, que es cerrado en $G$. Por el teorema 1.14 , esto implica que $G / H$ es un grupo regular. El grupo $G / H$ se llama grupo cociente y el mapeo $\pi: G \longrightarrow G / H$ es el homomorfismo cociente.

Las funciones cardinales se comportan mucho mejor en los grupos topológicos que en los espacios topológicos en general. Antes de presentar los resultados de funciones cardinales que necesitaremos para los capítulos posteriores, vamos a introducir una función cardinal que es exclusiva de grupos topológicos, el índice de acotación.

Un grupo $G$ es totalmente acotado si puede ser cubierto por un número finito de traslaciones de cualquier vecindad de la identidad. Es inmediato que todo grupo compacto es totalmente acotado, pues si $U$ es una vecindad de la identidad de $G$, entonces la familia $\{x U: x \in G\}$ es una cubierta abierta de $G$ que tiene una subcubierta finita por la compacidad de $G$. Claramente todo subgrupo de un grupo compacto es totalmente acotado, pero de hecho más es cierto: los grupos totalmente acotados son precisamente los subgrupos de los grupos compactos (ver [29]).

Guran en [14] generalizó la noción de ser totalmente acotado mediante la siguiente definición: 
DEFINICIÓN 1.15. Sea $\tau$ un cardinal infinito. Un grupo topológico $G$ es $\tau$-acotado si para toda vecindad $U$ de la identidad de $G$ existe un conjunto $K \subseteq G$ tal que $|K| \leq \tau$ y $G=K \cdot U$.

Es inmediato que los grupos $\sigma$-compactos y los grupos de Lindelöf son $\aleph_{0^{-}}$ acotados. Se puede verificar fácilmente (ver [14]) que los subgrupos de un grupo $\tau$-acotado, las imágenes continuas de un grupo $\tau$-acotado y los productos directos de grupos $\tau$-acotados son todos $\tau$-acotados.

El índice de acotación de un grupo topológico $G$ se define como el mínimo cardinal $\tau \geq \aleph_{0}$ tal que $G$ es $\tau$-acotado y se denota $i b(G)$.

$E$ l índice de acotación de un grupo $G$ sirve para relacionar a distintas funciones cardinales. El siguiente teorema, en su versión numerable, establece que todo grupo con la ccc es $\aleph_{0}$-acotado. Como todo grupo separable tiene la ccc, concluimos que todo subgrupo de un grupo separable es $\aleph_{0}$-acotado.

TeOREma 1.16. Todo grupo topológico $G$ satisface $i b(G) \leq c(G)$.

Demostración. Supongamos que $c(G)=\tau$ y que $G$ no es $\tau$-acotado. Entonces existe una vecindad $U$ de la identidad de $G$ tal que $K \cdot U \neq G$ para todo $K \subseteq G$ con $|K| \leq \tau$. Escojamos una vecindad abierta y simétrica $V$ de la identidad tal que $V^{2} \subseteq U$. Entonces podemos construir, por recursión, una sucesión $\left\{x_{\alpha}: \alpha<\tau^{+}\right\}$de puntos de $G$ de tal forma que $x_{\beta} \notin x_{\alpha} \cdot U$ para cualesquiera $\alpha, \beta$ con $\alpha<\beta<\tau^{+}$. Afirmamos que la familia de abiertos $\left\{x_{\alpha} V: \alpha<\tau^{+}\right\}$es disjunta. En efecto, si $\alpha<\beta<\tau^{+}$y $\left(x_{\alpha} V\right) \cap\left(x_{\beta} V\right) \neq \emptyset$, existen $v, w \in V$ tales que $x_{\alpha} v=x_{\beta} w$. Entonces $x_{\beta}=x_{\alpha} v w^{-1} \in\left(x_{\alpha} V\right) \cdot V^{-1}=$ $x_{\alpha} V^{2} \subseteq x_{\alpha} U$, lo cual contradice la manera en la que se construyó la sucesión $\left\{x_{\alpha}: \alpha<\tau^{+}\right\}$. Concluimos que $G$ es $\tau$-acotado y por lo tanto $i b(G) \leq \tau$.

Una consecuencia inmediata del teorema anterior, dado que $c(X) \leq d(X) \leq$ $w(X)$ para todo espacio topológico $X$, es la siguiente:

CoRolario 1.17. Para todo grupo topológico $G$ se cumplen las siguientes desigualdades:

$$
i b(G) \leq d(G) \text { y } i b(G) \leq w(G) .
$$

Con respecto a la celularidad en grupos topológicos se tiene el siguiente teorema de Tkachenko (ver [25]).

TEOREMA 1.18. Todo subgrupo de un grupo $\sigma$-compacto tiene celularidad numerable.

El índice de acotación es útil para evaluar el peso de un grupo topológico. $\mathrm{El}$ siguiente teorema tiene como consecuencia una igualdad entre funciones cardinales que es particular a la clase de grupos topológicos.

TEOREMA 1.19. Si $G$ es un grupo topológico, entonces $w(G)=i b(G) \cdot \chi(G)$. 
Demostración. Sabemos que $\chi(G) \leq w(G)$ y que $i b(G) \leq w(G)$, por lo tanto $i b(G) \cdot \chi(G) \leq w(G)$. Para la otra desigualdad, sea $\mathcal{A}$ una base local para la identidad de $G$ tal que $|\mathcal{A}| \leq \chi(G)$. Para cada conjunto $U \in \mathcal{A}$, sea $K_{U} \subseteq G$ con $\left|K_{U}\right| \leq i b(G)$ y tal que $K_{U} \cdot U=G$. Sea

$$
\mathcal{B}=\left\{x U: x \in K_{U} \text { y } U \in \mathcal{A}\right\} .
$$

Entonces $B$ es una familia de abiertos y $|\mathcal{B}| \leq i b(G) \cdot \chi(G)$. Afirmamos que $B$ es una base para $G$. En efecto, supongamos que $g \in G$ es arbitrario y tomemos $V \in \tau(g, G)$. Entonces existe una vecindad $U$ de la identidad tal que $g U \subseteq V$. Tomemos una vecindad de la identidad $W$ tal que $W^{-1} \cdot W \subseteq U$ y un elemento $B$ de $\mathcal{A}$ tal que $B \subseteq W$. Como $G=K_{B} \cdot U$, existe $x \in K_{B}$ tal que $g \in x B \subseteq g B^{-1} \cdot B \subseteq g W^{-1} \cdot W \subseteq g U \subseteq V$.

Corolario 1.20. Si $G$ es un grupo topológico, entonces $w(G)=d(G)$. $\chi(G)$.

Demostración. Todo espacio topológico $X$ satisface $d(X) \cdot \chi(X) \leq w(X)$. La otra desigualdad se sigue inmediatamente del teorema 1.19 y del corolario 1.17 .

Obsérvese que el corolario anterior no es válido para espacios compactos: la doble flecha de Alexandroff es un espacio compacto, primero numerable y separable, sin embargo $w(Z)=c$.

En la clase de los grupos topológicos tener carácter numerable implica la metrizabilidad del grupo. Este resultado es el teorema de Birkhoff-Kakutani (ver [3] y [19]). También se puede leer la demostración en [27] o [28].

TEOREMA 1.21. Un grupo topológico $G$ es metrizable si y sólo si $\chi(G)=\omega$.

Uno de los teoremas más notables acerca de los grupos $\tau$-acotados es el teorema del encaje de Guran (ver [14]), que caracteriza a los grupos $\tau$-acotados como subgrupos de un producto de grupos de peso $\leq \tau$. Para una demostración de este resultado también se pueden consultar [27] o [28]. El siguiente lema es crucial para la demostración del teorema del encaje de Guran.

LEMA 1.22. Sea $U$ una vecindad de la identidad en un grupo topológico $\tau$ acotado. Entonces existen un homomorfismo continuo y suprayectivo $\pi: G \longrightarrow$ $H$ de $G$ sobre un grupo topológico $H$ con $w(H) \leq \tau$ y una vecindad $V$ de la identidad de $H$ tales que $\pi^{-1}(V) \subseteq U$.

Con ayuda de este lema se puede dar una cota para la cardinalidad de los grupos topológicos en términos del índice de acotación y del pseudocarácter.

TEOREMA 1.23. Todo grupo topológico $G$ satisface $|G| \leq 2^{i b(G) \cdot \psi(G)}$.

Demostración. Sea $\tau=i b(G) \cdot \psi(G)$. Entonces existe una familia $\mathcal{A}=$ $\left\{U_{\alpha}: \alpha \in \tau\right\}$ de vecindades de la identidad $e$ de $G$ tal que $\cap \mathcal{A}=\{e\}$. Por el 
lema 1.22, para cada $\alpha \in \tau$ podemos encontrar un homomorfismo continuo y suprayectivo $\pi_{\alpha}: G \longrightarrow H_{\alpha}$ de $G$ sobre un grupo $H_{\alpha}$ con $w\left(H_{\alpha}\right) \leq \tau$ tal que $\pi_{\alpha}^{-1}\left(V_{\alpha}\right) \subseteq U_{\alpha}$ para alguna vecindad $V_{\alpha}$ de la identidad de $H_{\alpha}$.

Sea $\varphi: G \longrightarrow \prod_{\alpha \in \tau} H_{\alpha}$ el producto diagonal de la familia de homomorfismos $\left\{\pi_{\alpha}: \alpha \in \tau\right\}$. Sea $H=\varphi(G)$. Entonces claramente $w(H) \leq \tau$ y, dada la elección de $\mathcal{A}, \varphi: G \longrightarrow H$ es un homomorfismo continuo y biyectivo, es decir, un isomorfismo continuo. Como $|H| \leq 2^{w(H)}$ (ver el teorema 1.1), se sigue que $|G| \leq 2^{\tau}=2^{i b(G) \cdot \psi(G)}$.

\section{Uniones de cadenas}

El problema que nos ocupa en este trabajo es el siguiente. Supongamos que tenemos un espacio topológico $X$ expresado como unión de una cadena creciente de subespacios, es decir, $X=\bigcup\left\{X_{\alpha}: \alpha<\kappa\right\}$, donde $X_{\alpha} \subseteq X_{\beta}$ si $\alpha<\beta<\kappa$. ¿Qué se puede decir de $X$ si los valores de algunas funciones cardinales de los $X_{\alpha}$ 's están acotados? Mikhail Tkachenko $[\mathbf{2 3}, \mathbf{2 4}, 26]$ fue el primero en hacer un estudio sistemático de este problema, estudio continuado más tarde por Hajnal, Juhász y Szentmiklossy [16, 17].

Muchos son los resultados que se han obtenido para esta situación, pero aquí nos limitaremos a enunciar aquellos que involucran las funciones cardinales definidas anteriormente y que utilizaremos en los capítulos 2 y 3 .

Observemos primero que si $X=\bigcup\left\{X_{\alpha}: \alpha<\kappa\right\}$, donde $X_{\alpha} \subseteq X_{\beta}$ si $\alpha<\beta<\kappa$, entonces podemos suponer, sin perder generalidad, que $\kappa$ es un cardinal regular, pues si no lo es, podemos considerar una subcadena cofinal de longitud $c f(\kappa)$. La unión de tal subcadena es todo $X$ por ser cofinal y $c f(\kappa)$ es un cardinal regular. Obviamente también se puede suponer que si $\alpha<\beta<\kappa$, entonces $X_{\alpha} \subsetneq X_{\beta}$.

El primer resultado que presentamos se refiere al peso de red y es válido para cualquier espacio expresado como unión de subespacios, aunque éstos no formen una cadena.

TeOrema 1.24. Si $X=\bigcup\left\{X_{\alpha}: \alpha<\kappa\right\}$ y $n w\left(X_{\alpha}\right) \leq \lambda$ para toda $\alpha<\kappa$, entonces $n w(X) \leq \kappa \cdot \lambda$.

Demostración. Para cada $\alpha<\kappa$, sea $\mathcal{N}_{\alpha}$ una red para $X_{\alpha}$ tal que $\left|\mathcal{N}_{\alpha}\right| \leq$ $\lambda$. Consideremos a $\mathcal{N}=U\left\{\mathcal{N}_{\alpha}: \alpha<\kappa\right\}$. Claramente $\mathcal{N}$ es una red para $X$ y $|\mathcal{N}| \leq \kappa \cdot \lambda$.

El siguiente resultado establece que la celularidad de la unión no puede ser mucho más grande que la celularidad de los subespacios de la cadena. Para que los enunciados de los teoremas sean más concisos, en lo que sigue supondremos que el espacio $X=\bigcup\left\{X_{\alpha}: \alpha<\kappa\right\}$, donde $X_{\alpha} \subseteq X_{\beta}$ siempre que $\alpha \leq \beta<\kappa$.

TEOREMA 1.25. Si $c\left(X_{\alpha}\right)<\lambda$ para todo $\alpha<\kappa$, entonces $c(X) \leq \lambda$. Si además $\kappa>\lambda$, entonces $c(X)<\lambda$. 
Demostración. Dada una familia celular $\mathcal{G}$ en $X$ hagamos, para cada $\alpha<\kappa$,

$$
\mathfrak{S}_{\alpha}=\left\{G \in \mathcal{G}: G \cap X_{\alpha} \neq \emptyset\right\} .
$$

Claramente $\left|\mathfrak{S}_{\alpha}\right| \leq c\left(X_{\alpha}\right)<\lambda$. Más aún, si $\alpha<\beta<\kappa$, entonces $\mathfrak{S}_{\alpha} \subseteq \mathfrak{S}_{\beta} \mathrm{y}$ finalmente

$$
\mathcal{G}=\bigcup\left\{\mathfrak{S}_{\alpha}: \alpha<\kappa\right\}
$$

En consecuencia se tiene que $|\mathcal{G}| \leq \lambda$ y por lo tanto $c(X) \leq \lambda$. Si adicionalmente se tiene que $\lambda<\kappa$, entonces escojamos, para cada $G \in \mathcal{G}$, un ordinal $\alpha(G) \in \kappa$ tal que $G \in \mathcal{S}_{\alpha(G)}$. Dado que $\kappa$ es regular, existe $\alpha_{0} \in \kappa$ con $\alpha(G) \leq \alpha_{0}$ para todo $G \in \mathcal{G}$. Esto implica que $\mathcal{G}=\mathcal{S}_{\alpha_{0}}$ y por lo tanto $|\mathcal{S}| \leq c\left(X_{\alpha_{0}}\right)<\lambda$. Si $\lambda$ es un cardinal sucesor, esto implica inmediatamente que $c(X)<\lambda$. Si, por otro lado, $\lambda$ es un cardinal límite y $c(X)=\lambda$, podríamos escoger, para cada cardinal $\mu<\lambda$, una familia celular $\mathcal{G}^{\mu}$ en $X$ con $\left|\mathcal{G}^{\mu}\right|=\mu$ y luego encontrar, como lo hicimos anteriormente, $\alpha_{\mu}<\kappa$ tal que $\mathcal{G}_{\alpha_{\mu}}^{\mu}=\mathcal{G}^{\mu}$, es decir, tendríamos que para todo $G \in \mathcal{G}^{\mu}$ :

$$
G \cap X_{\alpha_{\mu}} \neq \emptyset \text {. }
$$

Pero nuevamente existiría un ordinal $\alpha \in \kappa$ tal que $\alpha_{\mu} \leq \alpha$ para toda $\mu<\lambda$, lo cual es imposible en virtud de que, por hipótesis, $c\left(X_{\alpha}\right)<\lambda$.

La idea para la demostración del siguiente resultado es exactamente la misma que para demostrar el teorema 1.24.

Teorema 1.26. Si $X$ es un espacio $T_{1}, p \in X$ y $\psi\left(p, X_{\alpha}\right) \leq \lambda$ cada vez que $p \in X_{\alpha}$, entonces $\psi(p, X) \leq \lambda \cdot \kappa$.

Demostración. Sea $p \in X$ y, para cada $\alpha \in \kappa$ tal que $p \in X_{\alpha}$, sea $\mathcal{B}_{\alpha}$ una familia de abiertos en $X$ de cardinalidad a lo sumo $\lambda$ y tal que $\{p\}=\bigcap\left\{B \cap X_{\alpha}\right.$ : $\left.B \in \mathcal{B}_{\alpha}\right\}$. Definamos ahora a $\mathcal{B}$ como $\mathcal{B}=\bigcup\left\{\mathcal{B}_{\alpha}: \alpha \in \kappa\right\}$. Entonces $|\mathcal{B}| \leq \lambda \cdot \kappa$ y $\{p\}=\cap B$. En efecto, si $q$ es cualquier otro punto de $X$, existe $\alpha \in \kappa$ tal que $p, q \in X_{\alpha}$. Por lo tanto existe $B \in \mathcal{B}_{\alpha}$ tal que $q \notin B \cap X_{\alpha}$, es decir, $q \notin B \mathrm{y}$, en consecuencia, $q \notin \cap B$.

Corolario 1.27. Si $\psi\left(X_{\alpha}\right) \leq \lambda$ para toda $\alpha<\kappa$, entonces $\psi(X) \leq \lambda \cdot \kappa$.

El siguiente teorema, aunque no es un resultado sobre cadenas, es importante en sí mismo y se usará en varias ocasiones en lo que sigue. Se puede pensar como un "teorema de reflejo" (reflexion theorem) sobre el peso, y tiene, en cierta forma, el sabor de los teoremas de cadenas, a saber, que una propiedad topológica del espacio está determinada por subespacios "chicos". Fue demostrado por Mikhail Tkachenko (ver [23]) para espacios regulares, la versión más general fue demostrada por Hajnal y Juhász en [16]. Vamos a presentar la demostración de la versión general, tal y como aparece en [18]. 
TEOREMa 1.28. Sean $X$ un espacio topológico arbitrario y $\kappa$ cualquier cardinal. Si $w(Y)<\kappa$ para todo subespacio $Y$ de $X$ de cardinalidad $\leq \kappa$, entonces $w(X)<\kappa$.

Antes de demostrar el teorema necesitamos introducir un nuevo concepto y probar un lema auxiliar. Dado un espacio topológico $X$ y un subespacio $S \subseteq X$, decimos que $S$ está separado por la izquierda si existe un buen orden < para $S$ tal que todo segmento final de $S$ bajo < es abierto en $S$. Nótese que si $|S|$ es un cardinal regular y $S$ está separado por la izquierda, entonces $d(S)=|S|$, pues todo conjunto denso es cofinal con respecto al buen orden <. Con ayuda de este concepto podemos enunciar el siguiente lema que es crucial para la demostración del teorema 1.28.

LEMA 1.29. Sea $X$ un espacio topológico. Supongamos que $\kappa$ es un cardinal regular tal que todo subespacio de $X$ separado por la izquierda tiene cardinalidad estrictamente menor que $\kappa$. Si $\left\{Y_{\alpha}: \alpha<\kappa\right\}$ es una cadena creciente de subespacios de $X$ con $Y=\bigcup\left\{Y_{\alpha}: \alpha<\kappa\right\}$ y $\mathcal{B}$ es una familia de abiertos de $X$ tal que para todo $\alpha<\kappa$

$$
\mathcal{B} \mid Y_{\alpha}=\left\{B \cap Y_{\alpha}: B \in \mathcal{B}\right\}
$$

es una base de $Y_{\alpha}$, entonces $\mathcal{B}\lceil Y$ es una base de $Y$.

Demostración. Supongamos que $B \backslash Y$ no es base de $Y$. Entonces existe un punto $y \in Y$ y un abierto $G \in \tau(y, Y)$ tal que para todo $B \in \mathcal{B}$ con $y \in B$ se verifica que $(B \cap Y) \backslash G \neq \emptyset$. Definimos por recursión una sucesión creciente de ordinales $\left\{\nu_{\alpha}: \alpha<\kappa\right\}$ y una sucesión $\left\{y_{\alpha}: \alpha<\kappa\right\}$ de puntos de $Y$ de tal forma que, para toda $\alpha<\kappa, y_{\alpha} \notin Y_{\nu_{\alpha}}$. Sea $\nu_{0}<\kappa$ tal que $y \in Y_{\nu_{0}}$. Como B $\mid Y_{\nu_{0}}$ es base de $Y_{\nu_{0}}$, existe $B_{0} \in \mathcal{B}$ tal que $y \in\left(B_{0} \cap Y_{\nu_{0}}\right) \subseteq G \cap Y_{\nu_{0}}$. Por la elección del punto $y$, podemos elegir $y_{0} \in\left(B_{0} \cap Y\right) \backslash G$. Note que entonces $y_{0} \notin Y_{\nu_{0}}$. Fijemos un $\alpha<\kappa$ y supongamos definidas a las sucesiones $\left\{y_{\beta}: \beta<\alpha\right\}$ y $\left\{\nu_{\beta}: \beta<\alpha\right\}$. Escojamos un ordinal $\nu_{\alpha}<\kappa$ mayor que todo $\nu_{\beta} \operatorname{con} \beta<\alpha$ y tal que $y_{\beta} \in Y_{\nu_{\alpha}}$ para todo $\beta<\alpha$. Esto es posible gracias a la regularidad de $\kappa$. Nuevamente existe $B_{\alpha} \in \mathcal{B}$ tal que $y \in B_{\alpha} \cap Y_{\nu_{\alpha}} \subseteq G \cap Y_{\nu_{\alpha}}$ y podemos elegir un punto $y_{\alpha} \in\left(B_{\alpha} \cap Y\right) \backslash G$.

Sólo resta observar que si $\beta<\alpha<\kappa$, entonces $y_{\beta} \notin B_{\alpha}$ pues como $y_{\beta} \in Y_{\nu_{\alpha}}$, esto implicaría que $y_{\beta} \in G \cap Y_{\nu_{\alpha}}$ y por construcción $y_{\beta} \notin G$. Pero entonces tenemos que todo segmento inicial del conjunto $S=\left\{y_{\alpha}: \alpha<\kappa\right\}$ es cerrado en $S$, es decir, que $S$ es un conjunto de cardinalidad $\kappa$ separado por la izquierda. Esto contradice una de las hipótesis del lema y concluye la demostración.

Ahora sí estamos en posibilidad de demostrar el teorema 1.28.

DEMOSTRACIÓN DEL TEOREMA 1.28. Supongamos primero que $\kappa$ es regular. Entonces claramente $X$ no puede tener un subespacio $S$ separado por la izquierda de cardinalidad $\kappa$, pues entonces $w(S) \geq d(S)=\kappa$ y estamos 
suponiendo que todo subespacio de $X$ de cardinalidad a lo sumo $\kappa$ tiene peso menor que $\kappa$. Vamos a definir, para cada $\alpha<\kappa$, un subconjunto $Y_{\alpha} \subseteq X$ con $\left|Y_{\alpha}\right|<\kappa$ y una familia de abiertos $\mathcal{B}_{\alpha}$ tal que $\left|\mathcal{B}_{\alpha}\right|<\kappa$. Hagamos $Y_{0}=\emptyset$ y $\mathcal{B}_{0}=\emptyset$. Supongamos que hemos definido $\left\{Y_{\beta}: \beta<\alpha\right\}$ y $\left\{\mathcal{B}_{\beta}: \beta<\alpha\right\}$ para algún $\alpha<\kappa$. Sea $Z_{\alpha}=\bigcup\left\{Y_{\beta}: \beta<\alpha\right\}$. Entonces $\left|Z_{\alpha}\right|<\kappa$ porque $\kappa$ es regular. Por lo tanto $w\left(Z_{\alpha}\right)<\kappa$ y podemos encontrar una familia $\mathcal{B}_{\alpha}$ de abiertos de $X$ tal que $\left|\mathcal{B}_{\alpha}\right|<\kappa, \cup\left\{\mathcal{B}_{\beta}: \beta<\alpha\right\} \subseteq \mathcal{B}_{\alpha}$ y $\mathcal{B}_{\alpha} \mid Z_{\alpha}$ es una base de $Z_{\alpha}$. Si $\mathcal{B}_{\alpha}$ es una base de $X$, hemos establecido que $w(X)<\kappa$ y paramos la construcción. Si no, entonces existe un punto $p_{\alpha} \in X$ y un conjunto abierto $G_{\alpha} \in \tau\left(p_{\alpha}, X\right)$ tal que para todo conjunto $B \in \mathcal{B}_{\alpha}$ tal que $p_{\alpha} \in B$, se tiene que $B \backslash G_{\alpha} \neq \emptyset$. Hagamos $\mathrm{C}_{\alpha}=\left\{B \in \mathcal{B}_{\alpha}: p_{\alpha} \in B\right\}$ y para cada $B \in \mathcal{C}_{\alpha}$ escojamos un punto $p(B) \in B \backslash G_{\alpha}$. Entonces definimos

$$
Y_{\alpha}=Z_{\alpha} \cup\left\{p_{\alpha}\right\} \cup\left\{p(B): B \in \mathbb{C}_{\alpha}\right\} .
$$

Claramente $\left|Y_{\alpha}\right|<\kappa$. Si la construcción se para en algún ordinal $\alpha<\kappa$, entonces $w(X)<\kappa$. Supongamos que la construcción continúa para todo $\alpha<\kappa$. Entonces podemos aplicar el lema 1.29 a la sucesión $\left\{Y_{\alpha}: \alpha<\kappa\right\}$ y a la familia $\mathcal{B}=\bigcup\left\{\mathcal{B}_{\alpha}: \alpha<\kappa\right\}$, porque por construcción $\mathcal{B}_{\alpha+1} \mid Y_{\alpha}$ es una base de $Y_{\alpha}$. El lema nos permite concluir que $B \mid Y$ es una base de $Y=U\left\{Y_{\alpha}: \alpha<\kappa\right\}$. Como $\left|Y_{\alpha}\right|<\kappa$ para todo ordinal $\alpha<\kappa$, tenemos que $|Y| \leq \kappa$ y, por hipótesis, $w(Y)<\kappa$. En consecuencia $B \mid Y$ tiene una subfamilia de cardinalidad igual a $w(Y)$ que también es base de $Y$. Como $\kappa$ es regular y $\mathcal{B}_{\alpha} \subseteq \mathcal{B}_{\beta}$ si $\alpha \leq \beta<\kappa$, concluimos que existe $\alpha<\kappa$ tal que $\mathcal{B}_{\alpha} \mid Y$ es una base de $Y$. Pero esto último es imposible, ya que $p_{\alpha} \in Y$ y por la construcción de $\mathcal{B}_{\alpha}, p_{\alpha}$ y $G_{\alpha}$, no existe $B \in \mathcal{B}_{\alpha}$ tal que $p_{\alpha} \in B \cap Y \subseteq G_{\alpha} \cap Y$, ya que si $p_{\alpha} \in B \in \mathcal{B}_{\alpha}$, entonces $p(B) \in Y_{\alpha} \cap\left(B \backslash G_{\alpha}\right)$. Por lo tanto la construcción tiene que parar en algún ordinal y, en consecuencia, $w(X)<\kappa$.

Supongamos ahora que $\kappa$ es un cardinal singular. Entonces en particular $\kappa$ es un cardinal límite. Afirmamos que en este caso existe un cardinal $\lambda<\kappa$ tal que $w(Y)<\lambda$ para todo $Y \subseteq X$ con $|Y| \leq \kappa$. En efecto, si no existe tal $\lambda$, escojamos, para cada $\lambda<\kappa$, un subespacio $Y_{\lambda} \subseteq X$ tal que $\left|Y_{\lambda}\right| \leq \kappa$ y $w\left(Y_{\lambda}\right) \geq$ $\lambda$. Definamos ahora a $Y=\bigcup\left\{Y_{\lambda}: \lambda<\kappa\right\}$. Entonces $|Y| \leq \kappa \cdot \kappa=\kappa$ y, como el peso es una función monótona (ver la seción 2), se tiene que $w(Y) \geq w\left(Y_{\lambda}\right) \geq \lambda$ para todo $\lambda<\kappa$. Esto implica que $w(Y) \geq \kappa$, lo cual es una contradicción. Sea $\lambda<\kappa$ tal que $w(Y)<\lambda$ para todo $Y \subseteq X$ con $|Y| \leq \kappa$. Nótese que entonces $w(Y)<\lambda^{+}$para todo $Y \subseteq X$ con $|Y| \leq \lambda^{+}$. Como $\lambda^{+}$es regular, podemos aplicar la primera parte de la demostración para concluir que $w(X)<\lambda^{+}<\kappa$.

El siguiente teorema de Mikhail Tkachenko (ver [23]) acota el peso de la unión si se tienen cotas para el peso de cada sumando. 
TeORema 1.30. Sea $X$ un espacio regular, $X=\bigcup\left\{X_{\alpha}: \alpha<\kappa\right\}$ donde $X_{\alpha} \subseteq X_{\beta}$ si $\alpha \leq \beta<\kappa$. Si $w\left(X_{\alpha}\right)<\lambda$ para todo $\alpha<\kappa$, entonces $w(X) \leq 2^{\lambda}$. Si además $\kappa>\lambda$, entonces $w(X)<\lambda$.

Demostración. Podemos suponer, sin perder generalidad, que $\kappa$ es regular. La demostración se divide en dos casos.

Caso 1. $\kappa \leq \lambda$. Sabemos, aplicando el primer inciso del teorema 1.2, que para todo $\alpha<\kappa, n w\left(X_{\alpha}\right) \leq w\left(X_{\alpha}\right)<\lambda$. Entonces el teorema 1.24 nos permite concluir que $n w(X) \leq \kappa \cdot \lambda=\lambda$. Sabemos que $d(X) \leq n w(X)$ (véase otra vez el teorema 1.2) por lo que, gracias al teorema 1.3, podemos concluir que $w(X) \leq 2^{d(X)} \leq 2^{n w(X)} \leq 2^{\lambda}$.

Caso 2. $\kappa>\lambda$. Sea $Y \subseteq X$ con $|Y| \leq \lambda$. Como $\lambda<\kappa$ y $\kappa$ es regular, existe un ordinal $\alpha<\kappa$ tal que $Y \subseteq X_{\alpha}$. Pero entonces $w(Y) \leq w\left(X_{\alpha}\right)<\lambda$. Hemos probado que todo subespacio de $X$ de cardinalidad a lo sumo $\lambda$ tiene peso estrictamente menor que $\lambda$. Aplicando el teorema 1.28 concluimos que $w(X)<\lambda$.

Es importante notar que el teorema anterior se puede refinar. Por ejemplo, en el primer caso basta con suponer que el peso de red de cada sumando es menor que $\lambda$ para obtener el resultado. Nótese también que con las mismas hipótesis se puede concluir que el peso de red de la unión es $\leq \lambda$. La versión que presentamos es justamente la que necesitamos en la demostración del teorema 3.6.

El último resultado de esta sección es un teorema de Tkachenko (ver [26]) que establece una cota para la estrechez de un espacio compacto si éste se expresa como unión de una cadena creciente de sus subespacios y se tienen cotas para la estrechez de cada sumando. La demostración que presentamos aquí es la que aparece en [18].

Teorema 1.31. Si $X$ es un espacio compacto, $X=\bigcup\left\{X_{\alpha}: \alpha<\kappa\right\}$ donde $X_{\alpha} \subseteq X_{\beta}$ si $\alpha \leq \beta<\kappa$ y $t\left(X_{\alpha}\right)<\lambda$ para cada $\alpha \in \kappa$, entonces $t(X) \leq \lambda$. Si además $\lambda<\kappa$, entonces $t(X)<\lambda$.

DEMOSTRACIón. Nuevamente suponemos que $\kappa$ es regular y dividimos la demostración en dos casos.

Caso 1. $\kappa \leq \lambda$. Por el teorema 1.6, basta probar que $F(X) \leq \lambda$, es decir, que $X$ no contiene ninguna sucesión libre de longitud $\lambda^{+}$. Supongamos por el contrario que $S=\left\{p_{\alpha}: \alpha<\lambda^{+}\right\}$es una sucesión libre. Como $\kappa \leq \lambda<\lambda^{+}$, existe $\alpha<\kappa$ tal que $\left|S \cap X_{\alpha}\right|=\lambda^{+}$. Por lo tanto podemos suponer que existe $\alpha<\kappa$ tal $S \subseteq X_{\alpha}$. De no ser así, podemos tomar una subsucesión adecuada. Sea $\mu=\lambda^{+}$. Para cada $\nu<\mu$, definamos a $F_{\nu}$ como la cerradura del segmento final de $S$ determinado por $\nu$, es decir, $F_{\nu}=\overline{\left\{p_{\alpha}: \nu \leq \alpha<\mu\right\}}$ y hagamos $F=\bigcap\left\{F_{\nu}: \nu<\mu\right\}$. Entonces $F \neq \emptyset$ porque $\left\{F_{\nu}: \nu<\mu\right\}$ es una familia decreciente de cerrados no vacíos y $X$ es compacto. Elijamos un punto $p \in F$. Como $S$ es libre, para cualquier $\nu<\mu$ se tiene que $p \notin \overline{\left\{p_{\alpha}: \alpha<\nu\right\}}$, es decir, 
$p \notin \bar{M}$ para todo conjunto $M \subseteq S$ con $|M|<\mu$. Sin embargo $p \in \bar{S}$. Sea $\beta<\kappa$ tal que $S \cup\{p\} \subseteq X_{\beta}$. Entonces $t\left(p, X_{\beta}\right) \geq t(p, S \cup\{p\}) \geq \mu=\lambda^{+}>\lambda$. Esto contradice la hipótesis acerca de la estrechez de los sumandos. Concluimos que $X$ no contiene ninguna sucesión libre de longitud $\lambda^{+}$y por lo tanto $t(X) \leq \lambda$.

Caso 2. $\kappa>\lambda$. Basta probar que $F(X)<\lambda$. Para cada cardinal regular $\mu \leq \lambda$, tal que $X$ contiene una sucesión libre de longitud $\mu$, escojamos una y llamémosla $S_{\mu}$. Note que, como $\mu \leq \lambda<\kappa, S_{\mu} \subseteq X_{\alpha}$ para algún ordinal $\alpha<\kappa$. Argumentando de la misma manera que en el caso anterior, escojamos, para cada $\mu$ tal, un punto $p_{\mu}$ análogo al punto $p$ del caso anterior. Como $\kappa$ es regular y cada $\mu$ considerada es estrictamente menor que $\kappa$, existe un ordinal $\beta<\kappa$ tal que $S_{\mu} \cup\left\{p_{\mu}\right\} \subseteq X_{\beta}$ para toda $\mu$. Tenemos entonces, igual que en el caso anterior, que $t\left(p_{\mu}, X_{\beta}\right) \geq \mu$ para toda $\mu$ tal. Por lo tanto $\mu \leq t\left(p_{\mu}, X_{\alpha}\right) \leq t\left(X_{\alpha}\right)$. En consecuencia, como $F(X)$ es el supremo de todos esos cardinales $\mu$, tenemos que $F(X) \leq t\left(X_{\alpha}\right)<\lambda$. 


\section{CAPÍTULO 2}

\section{Espacios diádicos y grupos localmente compactos}

En este capítulo estudiamos uniones de cadenas de subespacios de espacios compactos diádicos y de grupos topológicos localmente compactos. Como vimos en la sección 5 del capítulo 1, el problema general es el siguiente:

Sea $X$ un espacio topológico tal que $X=\bigcup\left\{X_{\alpha}: \alpha<\kappa\right\}$, donde $X_{\alpha} \subseteq X_{\beta}$ cada vez que $\alpha<\beta<\kappa$. ¿Qué se puede decir de $X$ si los valores de una función cardinal dada en los subespacios $X_{\alpha}$ están acotados? El objetivo de este capítulo es mejorar algunos de los resultados generales conocidos para el caso cuando $X$ es un espacio compacto diádico o un grupo localmente compacto.

Es bien conocido que un espacio compacto representable como la unión de una cadena de subespacios cerrados metrizables no tiene por qué ser metrizable y hasta puede tener estrechez no numerable (ver la sección 4). La situación cambia si el espacio es diádico, es decir, si es imagen continua del cubo generalizado de Cantor $D^{\mu}$ para alguna $\mu \geq \omega$. Vamos a probar, en los teoremas 2.2 y 2.8, que si un espacio diádico $X$ es la unión de una cadena de subespacios, cada uno de pseudocarácter numerable (o estrechez numerable), entonces $X$ es metrizable. Dado que todo grupo topológico localmente compacto es localmente diádico, por el teorema de Cleary-Morris [4], se tienen resultados similares para grupos localmente compactos; ver los teoremas 2.4 y 2.10. Los ejemplos de la sección 2 muestran que los teoremas 2.4 y 2.10 ya no son válidos si se reemplaza la compacidad local por la $\sigma$-compacidad o la compacidad numerable. Sin embargo, la conclusión sobre la metrizabilidad de un grupo $\sigma$-compacto o numerablemente compacto $G$ sigue valiendo si los subespacios $X_{\alpha}$ en la cadena creciente $G=\bigcup_{\alpha<\kappa} X_{\alpha}$ son primero-numerables y $c f(\kappa)>\omega_{1}$ (ver los corolarios 2.17 and 2.18).

Como siempre, denotamos con $w(X), \chi(X), \psi(X), t(X)$ y $c(X)$ el peso, el carácter, el pseudocarácter, la estrechez y la celularidad de un espacio $X$, respectivamente. El índice de acotación de un grupo topológico $G$ se denota por $i b(G)$. Finalmente, dado un cardinal infinito $\mu, A(\mu)$ denota la compactificación en un punto de un espacio discreto de cardinalidad $\mu$.

\section{Pseudocarácter y estrechez}

Hay muchos espacios compactos, primero numerables y no metrizables, como la doble flecha de Alexandroff y el círculo doble de Alexandroff. Por otro lado, los compactos diádicos primero numerables y los grupos topológicos 
primero numerables son metrizables en virtud de los teoremas de Esenin-Volpin y de Birkhoff-Kakutani, respectivamente (ver [12] y [3, 19]).

Estos resultados se pueden extender para cadenas crecientes de subespacios. En esta sección vamos a demostrar que para asegurar la metrizabilidad de un espacio diádico o de un grupo topológico localmente compacto, basta con expresarlos como uniones de cadenas crecientes de subespacios, cada uno de pseudocarácter numerable. Primero necesitamos un lema conocido en el folklor topológico.

LEMA 2.1. Sea $X$ un espacio compacto y $T_{2}$ expresado como la unión de una familia $\left\{X_{\alpha}: \alpha<\kappa\right\}$ de sus subespacios de tal forma que $\psi\left(X_{\alpha}\right)<\kappa$ para cada $\alpha<\kappa$, donde $\kappa$ es un cardinal regular infinito. Entonces existe un punto $x \in X$ tal que $\chi(x, X)<\kappa$.

DEMOSTRACión. Dado que por definición las funciones cardinales toman siempre valores infinitos, las hipótesis del lema implican que $\kappa>\omega$.

Supongamos, contrariamente a lo que queremos probar, que $\chi(x, X) \geq \kappa$ para todo $x \in X$. Vamos a definir, por recursión, una sucesión $\left\{F_{\alpha}: \alpha<\kappa\right\}$ de subconjuntos cerrados no vacíos de $X$ que satisfagan las siguientes condiciones para cada $\alpha<\kappa$ :

(1) $F_{\beta} \subseteq F_{\alpha}$ if $\alpha<\beta<\kappa$;

(2) $\chi\left(F_{\alpha}, X\right)<\kappa$;

(3) $F_{\alpha+1} \cap X_{\alpha}=\emptyset$.

Sea $F_{0}=X$. Supongamos que para algún $\alpha<\kappa$, hemos definido una sucesión $\left\{F_{\gamma}: \gamma<\alpha\right\}$ de subconjuntos cerrados no vacíos de $X$ de tal forma que se satisfacen las condiciones (1)-(3). Consideremos al conjunto $F=\bigcap_{\gamma<\alpha} F_{\gamma}$. Entonces $F$ es cerrado y no vacío. Por (2), para cada $\gamma<\alpha$, existe una familia $\mathcal{A}_{\gamma}$ de conjuntos abiertos de $X$ tal que $F_{\gamma}=\cap \mathcal{A}_{\gamma}$ y $\left|\mathcal{A}_{\gamma}\right|<\kappa$. Sea $\mathcal{A}=\bigcup_{\gamma<\kappa} \mathcal{A}_{\gamma}$. Entonces $F=\cap \mathcal{A}$ y, como $\kappa$ es regular y $\alpha<\kappa,|\mathcal{A}|<\kappa$. Por lo tanto $\psi(F, X)<\kappa$. Pero $X$ es compacto y $F$ es cerrado, por lo tanto $\psi(F, X)=\chi(F, X)<\kappa$. Si $\alpha$ es límite, definimos simplemente a $F_{\alpha}$ como $F$. Supongamos, pues, que $\alpha$ es sucesor, digamos que $\alpha=\nu+1$. Si $F \cap X_{\nu}=\emptyset$, de nuevo hacemos $F_{\alpha}=F$. Supongamos ahora que $F \cap X_{\nu} \neq \emptyset$ y escojamos un punto $p \in F \cap X_{\nu}$. Como $\psi\left(p, X_{\nu}\right)<\kappa$, existe una familia $\delta$ de abiertos en $X$ tal que $|\delta|<\kappa$ y $X_{\nu} \cap \cap \delta=\{p\}$. Puesto que $X$ es regular, podemos encontrar una familia $\tilde{\delta}$ de vecindades abiertas de $p$ en $X$ tal que $\delta \subseteq \tilde{\delta}$, cada miembro de $\tilde{\delta}$ contiene a la cerradura de otro miembro de $\tilde{\delta}$, y $|\tilde{\delta}| \leq|\delta| \cdot \omega<\kappa$.

Sea $K=F \cap \cap \tilde{\delta}$. Entonces $K$ es cerrado en $X, \chi(K, X)=\psi(K, X) \leq$ $\psi(F, X) \cdot|\tilde{\delta}|<\kappa$ y $K \cap X_{\nu}=\{p\}$. Por hipótesis, $\chi(x, X) \geq \kappa$ para todo $x \in X$, así que $K$ no es un conjunto unitario. Dado que $X$ es un espacio de Tychonoff, podemos encontrar un conjunto $K^{\prime} \subseteq K$ cerrado, no vacío y $G_{\delta}$ en $K$ tal que $p \notin K^{\prime}$. Claramente $K^{\prime} \cap X_{\nu}=\emptyset$. Definamos $F_{\alpha}=K^{\prime}$. Entonces $F_{\alpha} \cap X_{\nu}=\emptyset$ 
y $\chi\left(F_{\alpha}, X\right)=\psi\left(F_{\alpha}, X\right) \leq \omega \cdot \psi(K, X)<\omega \cdot \kappa=\kappa$. Por lo tanto la sucesión $\left\{F_{\gamma}: \gamma \leq \alpha\right\}$ satisface (1)-(3). Con esto termina nuestra construcción.

Sólo resta notar que el conjunto $P=\bigcap_{\alpha<\kappa} F_{\alpha}$ es no vacío y satisface $P \cap$ $X_{\alpha} \subseteq F_{\alpha+1} \cap X_{\alpha}=\emptyset$ para toda $\alpha<\kappa$, lo cual contradice la hipótesis de que $X=\bigcup\left\{X_{\alpha}: \alpha \in \kappa\right\}$.

Teorema 2.2. Sea $X$ un espacio diádico tal que $X=\bigcup\left\{X_{\alpha}: \alpha \in \kappa\right\}$, donde $X_{\alpha} \subseteq X_{\beta}$ si $\alpha<\beta<\kappa y \psi\left(X_{\alpha}\right) \leq \lambda$ para toda $\alpha \in \kappa$. Entonces $w(X) \leq \lambda$.

DEMOSTRACión. Supongamos, contrariamente a lo que queremos demostrar, que $w(X)>\lambda$. Por el teorema de Efimov-Gerlitz-Hagler, $X$ contiene una copia topológica de $D^{\mu}$, donde $\mu=\lambda^{+}$(ver el teorema 1.12). Entonces, claramente, $D^{\mu}=\bigcup_{\alpha<\kappa} Y_{\alpha}$, donde $Y_{\alpha}=X_{\alpha} \cap D^{\mu}$ para cada $\alpha<\kappa$. Tenemos, además, que $Y_{\alpha} \subseteq Y_{\beta}$ y $\psi\left(Y_{\alpha}\right) \leq \psi\left(X_{\alpha}\right) \leq \lambda$ si $\alpha<\beta<\kappa$. Podemos suponer, sin pérdida de generalidad, que $\kappa$ es un cardinal regular.

Caso 1. $\kappa \leq \lambda$. $D^{\mu}$ es la unión de una cadena de subespacios de pseudocarácter a lo sumo $\lambda$ y la cadena tiene longitud $\kappa$. El corolario 1.27 nos permite concluir que $\psi\left(D^{\mu}\right) \leq \kappa \cdot \lambda=\lambda$. Por lo tanto, $\mu=w\left(D^{\mu}\right)=\psi\left(D^{\mu}\right) \leq \lambda$. Pero $\mu=\lambda^{+}$, contradicción.

Caso 2. $\kappa>\lambda$. Igual que en el caso 1 , tenemos que $\mu=\psi\left(D^{\mu}\right) \leq \kappa \cdot \lambda=\kappa$, es decir, $\mu \leq \kappa$. Esto, junto con el hecho de que por definición $\mu=\lambda^{+}$, implica que $\lambda<\mu \leq \kappa$. Escojamos un punto $p \in D^{\mu}$. Como $\chi\left(p, D^{\mu}\right)=\mu>\lambda$, el teorema 1.11 garantiza la existencia de un conjunto $M \subseteq D^{\mu}$ tal que $M$ es discreto, $|M|=\mu$ y $M \cup\{p\}$ es homeomorfo a $A(\mu)$. Si $\mu<\kappa$, entonces, dado que $|M \cup\{p\}|=\mu<\kappa$ y $\kappa$ es regular, existe $\alpha<\kappa$ tal que $M \cup\{p\} \subseteq Y_{\alpha}$, y en consecuencia $\psi\left(p, Y_{\alpha}\right) \geq \psi(p, M \cup\{p\})=\mu>\lambda$. Esto contradice la desigualdad $\psi\left(Y_{\alpha}\right) \leq \lambda$. Por lo tanto $\mu=\kappa$. Tenemos que $D^{\kappa}=\bigcup_{\alpha<\kappa} Y_{\alpha}$, donde $\kappa$ es regular у $\psi\left(Y_{\alpha}\right) \leq \lambda<\kappa$ para toda $\alpha<\kappa$. Por el lema 2.1, existe un punto $x \in D^{\kappa}$ tal que $\chi\left(x, D^{\kappa}\right)<\kappa=\mu$. Esta contradicción demuestra que $w(X) \leq \lambda$.

Aplicando el teorema anterior con $\lambda=\omega$, se obtiene como corolario inmediato el siguiente teorema de metrización para espacios diádicos:

Corolario 2.3. Si un espacio diádico $X$ es la unión de una cadena creciente de subespacios, cada uno de pseudocarácter numerable, entonces $X$ es metrizable.

En virtud del teorema de Cleary-Morris [4], todo grupo localmente compacto es localmente diádico. Por lo tanto el teorema 2.2 tiene su contraparte para grupos localmente compactos.

TEOREMA 2.4. Si un grupo localmente compacto $G$ es la unión de una cadena de subespacios, cada uno de pseudocarácter a lo sumo $\lambda \geq \omega$, entonces $\chi(G) \leq \lambda$. 
Demostración. Como $G$ es localmente compacto, también es localmente diádico (ver [4]), por lo cual existe una vecindad abierta $U$ de la identidad $e$ en $G$ tal que la cerradura $\bar{U}$ es un espacio compacto diádico. Supongamos que $G$ es la unión de una cadena creciente de subespacios, cada uno de pseudocarácter a lo sumo $\lambda$. Aplicando el teorema 2.2 obtenemos $w(\bar{U}) \leq \lambda$ y, dado que todo grupo topológico es homogéneo (ver el teorema 1.13), tenemos que $\chi(G)=$ $\chi(e, G)=\chi(e, \bar{U}) \leq w(\bar{U}) \leq \lambda$.

El siguiente teorema de metrización para grupos localmente compactos es un corolario inmediato del teorema 2.4 .

CoROlario 2.5. Si un grupo localmente compacto es la unión de una cadena creciente de subespacios, cada uno de pseudocarácter numerable, entonces el grupo es metrizable.

Demostración. Por el teorema de Birkhoff-Kakutani, un grupo topológico es metrizable si y sólo si tiene carácter numerable. Sólo falta aplicar el teorema $2.4 \operatorname{con} \lambda=\omega$.

Nuestro siguiente resultado se refiere a la estrechez de espacios compactos diádicos. Por el teorema de Arhangel'skiì-Ponomarev en [2], el peso y la estrechez de un espacio diádico coinciden. Probaremos, en el teorema 2.8, que se puede dar una versión más fuerte de este resultado, una versión de "cadena creciente". Esta versión establece que si un espacio diádico es la unión de una cadena creciente de subespacios y la estrechez de cada sumando está acotada por un cardinal cualquiera, entonces el peso del espacio también está acotado por ese cardinal. Antes de probar esto, necesitamos dos lemas preliminares.

Recordemos que si $\tau>\omega$ es un cardinal regular, un subconjunto $J \subseteq \tau$ que es cofinal en $\tau$ y cerrado en $\tau$ con la topología inducida por el buen orden $\epsilon$ en $\tau$ se llama conjunto cerrado y no acotado. Si $S \subseteq \tau$ y $S \cap J \neq \emptyset$ para todo conjunto cerrado y no acotado $J$ en $\tau$, entonces $S$ es un conjunto estacionario. Si $\left\{J_{i}: i \in I\right\}$ es una familia de conjuntos cerrados y no acotados en $\tau$ y $|I|<\tau$, entonces $\cap\left\{J_{i}: i \in I\right\}$ también es un conjunto cerrado y no acotado en $\tau$. Si $\left\{S_{i}: i \in I\right\}$ es una familia de conjuntos no estacionarios de $\tau$ y $|I|<\tau$, entonces $\bigcup\left\{S_{i}: i \in I\right\}$ tampoco es estacionario (ver $\left.[8,21]\right)$.

LEMA 2.6. Sean $\tau>\omega$ un cardinal regular y $X$ cualquier conjunto no vacio. Si $X^{\tau}=\bigcup\left\{M_{\alpha}: \alpha<\tau\right\}$, entonces existen $\alpha_{0}<\tau$, un conjunto cerrado y no acotado $J$ en $\tau, y x^{*} \in X^{\tau \backslash J}$ tales que, para todo conjunto no estacionario $I \subseteq J, \pi_{I}\left(\pi_{\tau \backslash J}^{-1}\left(x^{*}\right) \cap M_{\alpha_{0}}\right)=X^{I}$, donde $\pi_{I} y \pi_{\tau \backslash J}$ son las proyecciones de $X^{\tau}$ a $X^{I}$ y a $X^{\tau \backslash J}$, respectivamente.

Demostración. Procedamos por reducción al absurdo y supongamos que el lema es falso. Entonces la afirmación del lema es falsa para todo conjunto cerrado y no acotado $J \subseteq \tau$ y todo $\alpha<\tau$, en particular para $J=\tau$ y $\alpha=0$. 
Por lo tanto existe un subconjunto no estacionario no vacío de $\tau$, digamos $I_{0}$, tal que $\pi_{I_{0}}\left(M_{0}\right) \neq X^{I_{0}}$. Escójase un punto $x_{0} \in X^{I_{0}}$ tal que $\pi_{I_{0}}^{-1}\left(x_{0}\right) \cap M_{0}=\emptyset$. Tomemos un ordinal $\beta<\tau$ arbitrario y supongamos que hemos definido familias $\left\{I_{\gamma}: \gamma<\beta\right\}$ y $\left\{x_{\gamma}: \gamma<\beta\right\}$ de tal forma que, para cualesquiera $\gamma, \gamma^{\prime}<\beta$ :

(1) $I_{\gamma} \subseteq \tau$ es no estacionario;

(2) si $\gamma \leq \gamma^{\prime}$, entonces $I_{\gamma} \subseteq I_{\gamma^{\prime}}$;

(3) $x_{\gamma} \in X^{I_{\gamma}}$

(4) si $\gamma \leq \gamma^{\prime}$, entonces $x_{\gamma^{\prime}} \mid I_{\gamma}=x_{\gamma}$;

(5) $\pi_{I_{\gamma}}^{-1}\left(x_{\gamma}\right) \cap M_{\gamma}=\emptyset$.

Si $\beta$ es un ordinal sucesor, digamos $\beta=\delta+1$, entonces, por (1), $I_{\delta}$ es no estacionario, y por lo tanto existe un conjunto cerrado y no acotado $F \subseteq \tau$ tal que $F \cap I_{\delta}=\emptyset$, es decir, $I_{\delta} \subseteq \tau \backslash F$. Por hipótesis, existe $I \subseteq F$ tal que $I$ es no estacionario y $X^{I} \neq \pi_{I}\left(\pi_{I_{\delta}}^{-1}\left(x_{\delta}\right) \cap M_{\delta+1}\right)$. Selecciónese un punto $z \in X^{I}$ tal que $\pi_{I}^{-1}(z) \cap \pi_{I_{\delta}}^{-1}\left(x_{\delta}\right) \cap M_{\delta+1}=\emptyset$. Entonces el conjunto $I_{\beta}=I \cup I_{\delta}$ es no estacionario, ya que tanto $I$ como $I_{\delta}$ lo son. Definamos a $x_{\beta}$ como la concatenación de $x_{\delta}$ con $z$. Como $\pi_{I_{\delta}}\left(x_{\beta}\right)=\pi_{I_{\delta}}\left(x_{\delta}\right)$ y $\pi_{I}\left(x_{\beta}\right)=\pi_{I}(z)$, tenemos que $\pi_{I_{\beta}}^{-1}\left(x_{\beta}\right) \cap M_{\delta+1}=\emptyset$. Por lo tanto, se satisfacen las condiciones (1)-(5).

Si $\beta$ es un ordinal límite, sean $L=\bigcup\left\{I_{\gamma}: \gamma<\beta\right\}$ y $x^{\prime}=\bigcup\left\{x_{\gamma}: \gamma<\beta\right\}$. Nótese que $x^{\prime} \in X^{L}$ y $L$ es no estacionario porque $\beta<\tau, \tau$ es regular y $I_{\gamma}$ es no estacionario para toda $\gamma<\beta$. Entonces podemos repetir el argumento anterior, con $L$ y $x^{\prime}$ en lugar de $I_{\delta}$ y $x_{\delta}$, respectivamente, para obtener un subconjunto no estacionario $I_{\beta}$ de $\tau$ y $x_{\beta} \in X^{I_{\beta}}$ que satisfagan las condiciones (1)-(5).

Hemos definido, por recursión, sucesiones $\left\{I_{\gamma}: \gamma<\tau\right\}$ y $\left\{x_{\gamma}: \gamma<\tau\right\}$ que satisfacen (1)-(5) para cualquier $\gamma<\tau$. Sean $I=\bigcup\left\{I_{\gamma}: \gamma<\tau\right\}$ y $x=\bigcup\left\{x_{\gamma}: \gamma<\tau\right\}$. Elijamos un elemento $x_{0} \in X$ y definamos $y \in X^{\tau}$ haciendo $y(\alpha)=x(\alpha)$ para toda $\alpha \in I$ y $y(\alpha)=x_{0}$ para toda $\alpha \notin I$. Entonces, para toda $\gamma<\tau$, tenemos que $\pi_{I_{\gamma}}(y)=x_{\gamma}$, es decir, $y \in \pi_{I_{\gamma}}^{-1}\left(x_{\gamma}\right)$, pero esto implica, por (5), que $y \notin M_{\gamma}$. Esto contradice la hipótesis de que $X^{\tau}=\bigcup\left\{M_{\gamma}: \gamma<\tau\right\}$.

LEMA 2.7. Supongamos que $X$ es un espacio topológico tal que $X^{\tau}=$ $\bigcup\left\{M_{\alpha}: \alpha<\tau\right\}$. Si $\alpha_{0}, J$ y $x^{*}$ son como en el lema 2.6, entonces, para todo conjunto no estacionario $I \subseteq J$, la restricción $\pi_{I}\left\lceil\pi_{\tau \backslash J}^{-1}\left(x^{*}\right) \cap M_{\alpha_{0}}\right.$ : $\pi_{\tau \backslash J}^{-1}\left(x^{*}\right) \cap M_{\alpha_{0}} \longrightarrow X^{I}$ es un mapeo abierto.

DEMOSTRACIÓN. Consideremos un conjunto no estacionario arbitrario $I \subseteq$ $J$. Sea $U=\left(\alpha_{1}, \ldots, \alpha_{n}, U_{1}, \ldots, U_{n}\right)=\bigcap\left\{p_{\alpha_{i}}^{-1}\left(U_{i}\right): i=1, \ldots, n\right\}$ un abjerto básico canónico de $X^{\tau}$. Probamos que $\pi_{I}\left(U \cap \pi_{\tau \backslash J}^{-1}\left(x^{*}\right) \cap M_{\alpha_{0}}\right)$ es abierto en $X^{I}$. Supongamos que $\alpha_{1}, \ldots, \alpha_{l} \in \tau \backslash J, \alpha_{l+1}, \ldots, \alpha_{k} \in J \backslash I$ and $\alpha_{k+1}, \ldots, \alpha_{n} \in I$. Sin pérdida de generalidad, podemos suponer que $x^{*}\left(\alpha_{i}\right) \in U_{i}$ para cada $i=1, \ldots, l$, 
pues de no ser así se tiene que $\pi_{I}\left(U \cap \pi_{\tau \backslash J}^{-1}\left(x^{*}\right) \cap M_{\alpha_{0}}\right)=\emptyset$. Probaremos que:

$$
\pi_{I}\left(U \cap \pi_{\tau \backslash J}^{-1}\left(x^{*}\right) \cap M_{\alpha_{0}}\right)= \begin{cases}\left(\alpha_{k+1}, \ldots, \alpha_{n}, U_{k+1}, \ldots, U_{n}\right) \subseteq X^{I} & \text { si } k<n ; \\ \pi_{I}\left(\pi_{\tau \backslash J}^{-1}\left(x^{*}\right) \cap M_{\alpha_{0}}\right)=X^{I}, & \text { si } k=n .\end{cases}
$$

Si $k<n$, consideremos cualquier punto $y \in X^{I}$ con $y\left(\alpha_{i}\right) \in U_{i}$ para $i=k+$ $1, \ldots, n$. Sea $I^{\prime}=I \cup\left\{\alpha_{l+1}, \ldots, \alpha_{k}\right\} \subseteq J$. Note que, dado que $I$ no es estacionario, $I^{\prime}$ tampoco lo es y, en consecuencia, $\pi_{I^{\prime}}\left(\pi_{\tau \backslash J}^{-1}\left(x^{*}\right) \cap M_{\alpha_{0}}\right)=X^{I^{\prime}}$. Tomemos una extensión $y^{\prime} \in X^{I^{\prime}}$ de $y$ tal que $y^{\prime}\left(\alpha_{i}\right) \in U_{i}$ para cada $i=l+1, \ldots, k$ y escojamos $z \in \pi_{\tau \backslash J}^{-1}\left(x^{*}\right) \cap M_{\alpha_{0}}$ de tal forma que $\pi_{I^{\prime}}(z)=y^{\prime}$. Entonces $z \in U \cap \pi_{\tau \backslash J}^{-1}\left(x^{*}\right) \cap M_{\alpha_{0}}$ y $\pi_{I}(z)=y$. La demostración para el caso $k=n$ es análoga.

Con este trabajo preliminar, estamos en posición de probar la generalización del teorema de Arhangel'skiir-Ponomarev.

Teorema 2.8. Sea $X$ un espacio diádico que se puede expresar como la unión de una cadena creciente de subespacios, cada uno de estrechez a lo sumo $\tau$, donde $\tau$ es cualquier cardinal infinito. Entonces $w(X) \leq \tau$.

Demostración. Supongamos que $X=\bigcup_{\alpha<\kappa} X_{\alpha}$, donde $\kappa$ y $\tau$ son cardinales infinitos, $t\left(X_{\alpha}\right) \leq \tau$ y $X_{\alpha} \subseteq X_{\beta}$ cuando $\alpha<\beta<\kappa$. Si $w(X)>\tau$, entonces, por el teorema de Efimov-Gerlitz-Hagler, $X$ contiene una copia topológica del cubo de Cantor $D^{\mu}$ con $\mu=\tau^{+}$. Entonces $D^{\mu}=\bigcup_{\alpha<\kappa} Y_{\alpha}$, donde $Y_{\alpha}=X_{\alpha} \cap D^{\mu}$ para cada $\alpha<\kappa$. Además, $t\left(Y_{\alpha}\right) \leq t\left(X_{\alpha}\right) \leq \tau$. Es suficiente, por lo tanto, probar que si $D^{\mu}=\bigcup_{\alpha<\kappa} Y_{\alpha}$, con $\kappa$ un cardinal regular, $Y_{\alpha} \subseteq Y_{\beta}$ si $\alpha<\beta<\kappa$ y $t\left(Y_{\alpha}\right) \leq \tau$ para cada $\alpha<\kappa$, entonces $\mu \leq \tau$. Supongamos, por el contrario, que $\mu>\tau$.

Caso 1. $\kappa>\mu$. En este caso tenemos a un espacio compacto que es unión de una cadena de subespacios, todos con estrechez $<\mu$. Como la longitud de la cadena es $\kappa>\mu$, podemos aplicar la segunda parte del teorema 1.31 y concluir que $t\left(D^{\mu}\right)<\mu$, lo cual es una contradicción (ver el teorema 1.10).

Caso 2. $\kappa \leq \mu$. Aplicando el lema 2.6, podemos encontrar $\alpha_{0}<\mu, J \subseteq \mu$, $J$ club, y $x^{*} \in D^{\mu \backslash J}$ tales que, para todo conjunto no estacionario $I \subseteq J$, $\pi_{I}\left(\pi_{\mu \backslash J}^{-1}\left(x^{*}\right) \cap Y_{\alpha_{0}}\right)=D^{I}$. Sea $I \subseteq J$ un conjunto no estacionario con $|I|=\mu$. Entonces, por el lema 2.7, $\pi_{I} \uparrow \pi_{\mu \backslash J}^{-1}\left(x^{*}\right) \cap Y_{\alpha_{0}}: \pi_{\mu \backslash J}^{-1}\left(x^{*}\right) \cap Y_{\alpha_{0}} \longrightarrow D^{I}$ es un mapeo abierto. Como los mapeos abiertos no elevan la estrechez (véase el teorema 1.5), tenemos lo siguiente:

$$
\mu=|I|=t\left(D^{I}\right) \leq t\left(\pi_{\mu \backslash J}^{-1}\left(x^{*}\right) \cap Y_{\alpha_{0}}\right) \leq t\left(Y_{\alpha_{0}}\right) \leq \tau .
$$

Por lo tanto, $\mu \leq \tau$, contradicción. Esto completa la demostración.

COROlario 2.9. Si un espacio diádico es unión de una cadena creciente de subespacios, todos con estrechez numerable, entonces el espacio es metrizable. 
Demostración. En el teorema 2.8 tómese $\lambda=\omega$. Concluimos que el espacio es segundo numerable $\mathrm{y}$, por lo tanto, metrizable.

Otra vez, el teorema 2.8 tiene su análogo para grupos localmente compactos.

TEOREMA 2.10. Si un grupo localmente compacto $G$ es la unión de una cadena de subespacios, cada uno de estrechez $\leq \tau$, entonces $\chi(G) \leq \tau$.

Demostración. Por el teorema de Cleary-Morris ([4]), existe una vecindad abierta $U$ de la identidad $e$ en $G$ tal que la cerradura $K=\bar{U}$ es un espacio compacto diádico. Supongamos que $G$ es la unión de una cadena de sus subespacios, cada uno de estrechez a lo sumo $\tau$. Entonces el teorema 2.8 implica que $w(K) \leq \tau$, por lo cual $\chi(G)=\chi(e, G)=\chi(e, K) \leq w(K) \leq \tau$.

COROLARIO 2.11. Si un grupo localmente compacto $G$ es la unión de una cadena de subespacios de estrechez numerable, entonces $G$ es metrizable.

Demostración. Aplicando el teorema 2.10 con $\tau=\omega$, obtenemos que $G$ tiene carácter numerable y es, por lo tanto, metrizable.

\section{Algunos ejemplos}

Los teoremas 2.4 y 2.10 fueron enunciados para grupos topológicos localmente compactos. Es natural preguntarse si pueden extenderse a otras clases de grupos topológicos. En esta sección se presentan ejemplos que demuestran que los teoremas mencionados no son válidos ni para grupos numerablemente compactos ni para grupos $\sigma$-compactos. Más aún, en los ejemplos 2.13 y 2.15 se muestra que existen grupos numerablemente compactos y grupos $\sigma$-compactos que son uniones de cadenas crecientes de subespacios segundo numerables y cuya estrechez es no numerable.

Antes de dar los ejemplos, recordemos algunas definiciones.

Supongamos que $\left\{X_{i}: i \in I\right\}$ es una familia de espacios topológicos y $a=\left(a_{i}\right)_{i \in I}$ es un punto del producto $\Pi\left\{X_{i}: i \in I\right\}$. El $\Sigma$-producto de la familia con respecto al punto $a$ está dado por

$$
\Sigma(a)=\left\{x \in \prod_{i \in I} X_{i}: \mid\left\{i \in I: x_{i} \neq a_{i}\right\} \leq \omega\right\}
$$

y el $\sigma$-producto por

$$
\sigma(a)=\left\{x \in \prod_{i \in I} X_{i}: \mid\left\{i \in I: x_{i} \neq a_{i}\right\}<\omega\right\} .
$$

Si $x \in \Pi\left\{X_{i}: i \in I\right\}$ es un punto arbitrario, el soporte de $x$ con respecto a $a$ es $\operatorname{supp}(x)=\left\{i \in I: x_{i} \neq a_{i}\right\}$.

EJEMPLO 2.12. Considérese el $\Sigma$-producto de $\omega_{1}$ copias de $D$ :

$$
\Sigma=\left\{x \in D^{\omega_{1}}:\left|\left\{\alpha<\omega_{1}: x_{\alpha} \neq 0\right\}\right| \leq \omega\right\} .
$$


Directamente de la definición de la topología del producto se verifica que $\Sigma$ es un subgrupo denso de $D^{\omega_{1}}$. Ahora probamos que $\Sigma$ es numerablemente compacto. Para esto consideremos un subconjunto infinito numerable $F \subseteq \Sigma$. Tenemos que demostrar que $F$ tiene un punto de acumulación en $\Sigma$. Sea $H=$ $\bigcup\{\operatorname{supp}(x): x \in F\}$, donde, para cada $x \in D^{\omega_{1}}, \operatorname{supp}(x)=\left\{\alpha \in \omega_{1}: x_{\alpha} \neq 0\right\}$. Por definición, para cada $x \in F, \operatorname{supp}(x)$ es numerable y por lo tanto $H$ es un conjunto numerable. Consideremos el producto $G=\prod_{\alpha \in H} D \times \overline{0}$, donde $\overline{0}=\prod_{\alpha \in \omega_{1} \backslash H}\{0\}$ es el 'cero' de $D^{\omega_{1} \backslash H}$. Observe que $G$ es compacto y $F \subseteq G$, por lo que $F$ tiene un punto de acumulación en $G$. Como $G \subseteq \Sigma, F$ tiene un punto de acumulación en $\Sigma$ y queda probado que $\Sigma$ es numerablemente compacto. Como $\Sigma$ es denso en $D^{\omega_{1}}, \chi(x, \Sigma)=\chi\left(x, D^{\omega_{1}}\right)=\omega_{1}$ para todo $x \in \Sigma$, y por lo tanto $\chi(\Sigma)=\omega_{1}$. De hecho se puede verificar de manera inmediata que $\psi(\Sigma)=\omega_{1}$. Además, $\Sigma=\bigcup\left\{X_{\alpha}: \alpha<\omega_{1}\right\}$, donde

$$
X_{\alpha}=\left\{x \in D^{\omega_{1}}:\left\{\beta<\omega_{1}: x_{\beta} \neq 0\right\} \subseteq \alpha\right\}
$$

para toda $\alpha<\omega_{1}$ y $X_{\alpha} \subseteq X_{\beta}$ si $\alpha<\beta<\omega_{1}$. Es fácil ver que cada $X_{\alpha}$ es un subgrupo compacto metrizable de $D^{\omega_{1}}$, pues es topológicamente isomorfo a $D^{\omega}$. Tenemos, por lo tanto, que $w\left(X_{\alpha}\right)=\chi\left(X_{\alpha}\right)=\psi\left(X_{\alpha}\right)=t\left(X_{\alpha}\right)=\omega$ para cada $\alpha<\omega_{1}$, y sin embargo el grupo $\Sigma$ no es metrizable. Note que el grupo $\Sigma$ es de Fréchet-Urysohn, es decir, para todo $A \subseteq \Sigma$ y todo $x \in \bar{A}$, existe una sucesión $\left\{x_{n}: n \in \omega\right\}$ de puntos de $\Sigma$ que converge a $x$ (ver [11]). Esto implica que $t(\Sigma)=\omega$.

Vamos a modificar ligeramente esta construcción en el ejemplo 2.13 para obtener un grupo $G$ numerablemente compacto similar, pero esta vez con $t(G)=\omega_{1}$.

EJEMPLO 2.13. Como en ejemplo 2.12, consideremos el subgrupo $\Sigma \subseteq D^{\omega_{1}}$. Tómese $p \in D^{\omega_{1}} \backslash \Sigma$ y defínase el grupo $G=\langle\Sigma \cup\{p\}\rangle$, el subgrupo de $D^{\omega_{1}}$ generado por $\Sigma \cup\{p\}$. Entonces $G$ es numerablemente compacto y no metrizable. Como $p \notin \Sigma, p$ tiene soporte de cardinalidad $\omega_{1}$. Si $A \subseteq \Sigma$ es numerable, entonces el conjunto $I=\bigcup\{\operatorname{supp}(x): x \in A\}$ es numerable, por lo que existe un ordinal $\xi \in \operatorname{supp}(p) \backslash I$. Entonces $U=\pi_{\xi}^{1}(\{1\})$ es un abierto que contiene a $p$ y no intersecta a $A$. Esto demuestra que $t(G)=t(p, G)=\omega_{1}$. Para cada $\alpha<\omega_{1}$, definamos $Y_{\alpha}$ como $\left\langle X_{\alpha} \cup\{p\}\right\rangle$, con $X_{\alpha}$ igual al del ejemplo 2.12. Entonces $G$ es la unión de la cadena $\left\{Y_{\alpha}: \alpha<\omega_{1}\right\}$ y cada sumando de la cadena tiene peso numerable.

EJEMPLO 2.14. En $D^{\omega_{1}}$, consideremos el $\sigma$-producto:

$$
G=\left\{x \in D^{\omega_{1}}:\left|\left\{\alpha<\omega_{1}: x_{\alpha} \neq 0\right\}\right|<\omega\right\} .
$$

Entonces $G$ es un subgrupo $\sigma$-compacto y denso de $D^{\omega_{1}}$. La densidad de $G$ se sigue inmediatamente de la definición de la topología del producto. Para ver que $G$ es $\sigma$-compacto definimos, para cada $n \in \mathbb{N}$ :

$$
G_{n}=\left\{x \in D^{\omega_{1}}:|\operatorname{supp}(x)| \leq n\right\} \text {. }
$$


Entonces claramente $G_{n} \subseteq G$ para toda $n \in \mathbb{N}$ y $G=\bigcup_{n \in \mathbb{N}} G_{n}$. Por lo tanto basta probar que cada $G_{n}$ es compacto, esto lo haremos probando que cada $G_{n}$ es cerrado en $D^{\omega_{1}}$. Sea $p \in D^{\omega_{1}} \mid G_{n}$. Entonces $|\operatorname{supp}(p)|>n$, por lo que existen $\alpha_{1}, \ldots, \alpha_{n+1} \in \omega_{1}$ tales que $x_{\alpha_{i}} \neq 0$ para toda $i=1, \ldots, n+1$. Si hacemos $U_{i}=\{1\}$ para cada $i$, entonces $U=\bigcap_{i=1}^{n+1} \pi_{\alpha_{i}}^{-1}\left(U_{i}\right)$ es abierto en $D^{\omega_{1}}, p \in U$ y $G_{n} \cap U=\emptyset$. Concluimos que $G_{n}$ es cerrado en $D^{\omega_{1}}$ y es, en consecuencia, compacto. Claramente $\chi(G)=\chi\left(D^{\omega_{1}}\right)=\omega_{1}$ y por lo tanto $G$ no es metrizable. Para cada $\alpha<\omega_{1}$, sea

$$
G_{\alpha}=\{x \in G: \operatorname{supp}(x) \subseteq \alpha\} .
$$

Entonces $G_{\alpha} \subseteq G_{\beta}$ si $\alpha \leq \beta$ y $G=\bigcup\left\{G_{\alpha}: \alpha<\omega_{1}\right\}$. Además, cada $G_{\alpha}$ tiene peso numerable. Sin embargo, el grupo $\sigma$-compacto $G$ no es metrizable.

EJEMPLO 2.15. Para obtener un grupo $\sigma$-compacto con estrechez no numerable que sea la unión de una cadena creciente de subespacios de peso numerable, consideramos el $\sigma$-producto en $D^{\omega_{1}}$, como en 2.14, y tomamos $p \in D^{\omega_{1}} \backslash \Sigma$, para repetir la construcción del ejemplo 2.13 .

\section{Grupos totalmente acotados y $\sigma$-compactos}

En los ejemplos 2.12 - 2.15 la longitud de las cadenas es $\omega_{1}$. En esta sección demostramos que cuando la cadena es más larga, la situación cambia.

Recordemos que los subgrupos de los grupos topológicos $\sigma$-compactos se llaman $\sigma$-precompactos. Se sabe que todo subgrupo de un grupo topológico $\sigma$-compacto tiene celularidad numerable (ver [25] y el teorema 1.18).

TEOREMA 2.16. Sea $G$ un grupo topológico $\sigma$-precompacto tal que $G=$ $\bigcup\left\{X_{\alpha}: \alpha<\kappa\right\}$, donde $c f(\kappa)>\omega_{1}$ y $X_{\alpha} \subsetneq X_{\beta}$ cuando $\alpha<\beta<\kappa$. Si cada $X_{\alpha}$ es primero numerable, entonces $G$ es segundo numerable.

Demostración. Sea $H$ es un subgrupo de $G$ de cardinalidad a lo sumo $\omega_{1}$. Entonces, dado que $G$ es $\sigma$-precompacto, podemos aplicar un resultado de Tkachenko (ver [25]) para concluir que $H$ tiene celularidad numerable. Además, como $c f(\kappa)>\omega_{1}$, se sigue que $H \subseteq X_{\alpha}$ para alguna $\alpha<\kappa$, así que $\chi(H) \leq$ $\chi\left(X_{\alpha}\right)=\omega$. El teorema 1.16 afirma que el índice de acotación de un grupo es menor o igual a su celularidad, por lo que tenemos que $i b(H) \leq c(H)=\omega$, es decir, el grupo $H$ es $\aleph_{0}$-acotado. Ahora podemos aplicar el teorema 1.19 y concluir que

$$
w(H)=i b(H) \cdot \chi(H)=\omega \cdot \omega=\omega .
$$

Hemos establecido que todo subgrupo de $G$ de cardinalidad a lo sumo $\omega_{1}$ tiene peso numerable. Tomemos ahora cualquier subconjunto $Y \subseteq G$ con $|Y| \leq \omega_{1}$ y consideremos el subgrupo $H$ de $G$ generado por $Y$. Entonces $|H| \leq \omega_{1}$, y por lo tanto $w(Y) \leq w(H)=\omega$. En resumen, todo subespacio de $G$ de cardinalidad a lo sumo $\omega_{1}$ tiene peso numerable. Concluimos, aplicando el teorema 1.28, que $w(G)=\omega$. 
El siguiente corolario es inmediato.

Corolario 2.17. Sea $G$ un grupo topológico $\sigma$-compacto, $G=\bigcup_{\alpha<\kappa} X_{\alpha}$. donde $c f(\kappa)>\omega_{1}$ y $X_{\alpha} \subsetneq X_{\beta}$ si $\alpha<\beta<\kappa$. Si cada $X_{\alpha}$ es primero numerable, entonces $G$ es segundo numerable.

Los grupos totalmente acotados son precisamente los subgrupos de los grupos topológicos compactos, por lo que también el siguiente corolario es inmediato.

COROLARIO 2.18. Sea $G$ un grupo topológico totalmente acotado, $G=$ $\bigcup_{\alpha<\kappa} X_{\alpha}$, donde $c f(\kappa)>\omega_{1}$ y $X_{\alpha} \subsetneq X_{\beta}$ cuando $\alpha<\beta<\kappa$. Si cada $X_{\alpha}$ es primero numerable, entonces $G$ es segundo numerable.

\section{Observaciones finales}

Los resultados de este capítulo dependen fuertemente del hecho de que los espacios considerados son compactos diádicos o grupos topológicos. No pueden ser generalizados a la clase de los espacios compactos. La doble flecha de Alexandroff y el círculo doble de Alexandroff son espacios compactos, primero numerables y de peso $c$, por lo que no son metrizables.

El siguiente ejemplo muestra que los resultados de la sección 1 no son válidos ni siquiera para espacios compactos linealmente ordenados.

EJEMPLO 2.19. Sea $W$ el espacio de todos los ordinales menores o iguales al primer ordinal no numerable $\omega_{1}$ con la topología inducida por el buen orden natural en $W$ (ver [11]). $W$ con esta topología es un espacio compacto linealmente ordenado.

Nótese que $W=\omega_{1} \cup\left\{\omega_{1}\right\}$, por lo que $W=U\left\{X_{\alpha}: \alpha<\omega_{1}\right\}$, donde $X_{\alpha}=\alpha \cup\left\{\omega_{1}\right\}$ para todo $\alpha<\omega_{1}$. Esta es una cadena creciente de subespacios cerrados, y por lo tanto compactos, de $W$. Para cada $\alpha<\omega_{1}$ se tiene que $t\left(X_{\alpha}\right)=\psi\left(X_{\alpha}\right)=\chi\left(X_{\alpha}\right)=\omega$.

Por último, como $\omega_{1}$ es regular, no tiene sucesiones cofinales de longitud $\omega$. Esto implica que $\psi\left(\omega_{1}, W\right)=\chi\left(\omega_{1}, W\right)=\omega_{1}$. Tenemos por lo tanto que $w(W) \geq \omega_{1}$ y que $W$ no es metrizable.

Este ejemplo demuestra que ninguno de los resultados de la sección 1 puede ser extendido a la clase de los espacios compactos linealmente ordenados. 


\section{CAPÍTULO 3}

\section{Cadenas de subgrupos}

\section{Introducción}

En este capítulo consideramos un caso más especíico, ahora vamos a considerar a grupos topológicos que son uniones de cadenas crecientes de subgrupos, no simplemente de subespacios. Tenemos la situación siguiente: $G$ es un grupo topológico y $G=\bigcup\left\{G_{\alpha}: \alpha \in \kappa\right\}$, donde $G_{\alpha} \leqslant G_{\beta} \leqslant G$ si $\alpha<\beta<\kappa$. Nos interesa saber qué se puede decir acerca de $G$ si conocemos los valores de algunas funciones cardinales en los $G_{\alpha}$ 's. En la sección 2 probaremos que si el índice de acotación de todos los subgrupos de la cadena es estrictamente menor que $\lambda$ para algún cardinal infinito $\lambda$, entonces el índice de acotación de $G$ es a lo sumo $\lambda$. Probaremos también que si tanto el carácter como el índice de acotación de cada $G_{\alpha}$ son estrictamente menores que $\lambda$, entonces el peso de $G$ es menor o igual a $2^{\lambda}$. Por último probaremos que las hipótesis de este último resultado se pueden debilitar, pero sólo si la longitud de la cadena, $\kappa$, no es igual a $\lambda^{+}$.

Se sabe que todo grupo topológico $G$ satisface la desigualdad $|G| \leq 2^{i b(G) \cdot \psi(G)}$ (ver el teorema 1.23). En la sección 3 probamos una versión de este resultado en términos de cadenas crecientes para grupos numerablemente compactos y construimos un ejemplo que demuestra que esta generalización no es válida en general, ni siquiera para grupos pseudocompactos.

Escribimos $H \leqslant G$ cuando $H$ es un subgrupo de $G$ y $H \triangleleft G$ cuando $H$ es un subgrupo normal de $G$. El índice de acotación del grupo $G$ (ver la definición 1.15) se denota $i b(G)$. La cardinalidad del continuo se denota c, es decir, $\mathfrak{c}=2^{\aleph_{0}}$. En lo que sigue todos los grupos considerados son de Hausdorff, lo que implica, en virtud del teorema 1.14, que son regulares.

\section{Indice de acotación}

Antes de probar los resultados principales de esta sección, presentamos tres hechos simples acerca de esta función cardinal. El primero de ellos es de Guran (ver [14]).

Proposición 3.1. Si $H$ es un subgrupo denso de $G$, entonces $i b(H)=$ $i b(G)$.

Demostración. Para ver que $i b(H) \leq i b(G)$, sea $U$ una vecindad de la identidad $e$ de $H$. Existen vecindades $V$ y $W$ de $e$ en $G$ tales que $U=V \cap H$ y $W^{-1} \cdot W \subseteq U$. Entonces existe $K \subseteq G$ con $|K| \leq i b(G)$ tal que $K \cdot W=G$. 
Si $x \in K$ y $x W \cap H \neq \emptyset$, tomamos un punto $a_{x} \in x W \cap H$. Si no es así, hacemos $a_{x}=e$. Claramente $a=\left\{a_{x}: x \in K\right\}$ es un subconjunto de $H$ de cardinalidad $\leq i b(G)$. Probamos que $H=A \cdot U$. Para esto, sea $h \in H$. Como $G=K \cdot W$, existen $x \in K$ y $w \in W$ tales que $h=x \cdot w$. Por lo tanto $a_{x} \in H \cap x W$, es decir, $a_{x}=x \cdot w_{0}$, para algún $w_{0} \in W$. En consecuencia $h=x \cdot w=x \cdot w_{0} \cdot w_{0}^{-1} \cdot w=a_{x} \cdot w_{0}^{-1} \cdot w_{0} \in A \cdot W^{-1} \cdot W \subseteq A \cdot U$.

Para la otra desigualdad basta probar que, para cualquier $\tau$, si $H$ es $\tau$ acotado, también lo es $G$. Dada cualquier vecindad abierta $U$ de la identidad $e$ en $G$, escojamos una vecindad abierta y simétrica $V$ de $e$ en $G$ tal que $V^{2} \subseteq U$. Sea $W=V \cap H$. Como $H$ es $\tau$-acotado, existe un subconjunto $K \subseteq H$ tal que $|K| \leq \tau$ y $K \cdot W=H$. Afirmamos que $K \cdot U=G$. En efecto, si $x \in G$, entonces $x \in \bar{H}$ y por lo tanto $x V \cap H \neq \emptyset$. En consecuencia existe $k \in K$ tal que $x V \cap k W \neq \emptyset$. Por lo tanto $x \in k W \cdot V^{-1} \subseteq k V^{2} \subseteq k U$ y tenemos que $G=K \cdot U$.

Si $N$ es un subgrupo normal y cerrado de $G$, entonces podemos considerar al grupo cociente $G / N$. Como $N<G$ y $G / N$ es imagen homomorfa de $G$, sus índices de acotación son menores o iguales al índice de acotación de $G$. El siguiente resultado establece que no pueden ser ambos estrictamente menores que $i b(G)$.

Proposición 3.2. Si $N \triangleleft G$, entonces $i b(G)=i b(N) \cdot i b(G / N)$.

Demostraclón. Note que $i b(N) \leq i b(G)$ y que $i b(G / N) \leq i b(G)$. Dado cualquier cardinal infinito $\tau$, supongamos que tanto $N$ como $G / N$ son $\tau$ acotados. Dada cualquier vecindad abierta $U$ de la identidad $e$ en $G$, escojamos una vecindad abierta $V$ de $e$ tal que $V^{2} \subseteq U$ y hagamos $W=V \cap N$. Entonces existen conjuntos $K \subseteq N$ y $L \subseteq G$, ambos de cardinalidad a lo sumo $\tau$, tales que $N=K \cdot W$ y $G / N=L^{*} \cdot V^{*}$ donde, para cualquier $A \subseteq G$, $A^{*}=\{N x: x \in A\}$. Afirmamos que $G=L \cdot K \cdot U$. Para ver esto, observe que dado cualquier $g \in G$, existen $l \in L$ y $v \in V$ tales que $N g=N l \cdot N v$. Por lo tanto, $g \in N l v=l N v \subseteq L \cdot N \cdot V=L \cdot K \cdot W \cdot V \subseteq L \cdot K \cdot V^{2} \subseteq L \cdot K \cdot U$. Como $|L \cdot K| \leq \tau$, concluimos que $i b(G) \leq \tau$.

El siguiente resultado proporciona información acerca del comportamiento del índice de acotación en los subgrupos de un grupo topológico.

Proposición 3.3. Si $G$ es un grupo topológico con $i b(G)=\tau$, entonces, para todo cardinal infinito $\lambda<\tau$, existe un subgrupo $H \leqslant G$ tal que $|H|=$ $i b(H)=\lambda$.

Demostración. Considérese cualquier $\lambda<\tau$. Entonces, como $i b(G)>\lambda$, existe una vecindad $U$ de la identidad de $G$ tal que $K \cdot U \neq G$ para todo conjunto $K \subseteq G$ tal que $|K| \leq \lambda$. Por lo tanto podemos construir, por recursión, una sucesión de puntos de $G,\left\{x_{\alpha}: \alpha<\lambda\right\}$, de tal forma que, para toda $\alpha<\lambda$, $x_{\alpha} \notin\left\{x_{\beta}: \beta<\alpha\right\} \cdot U$. Sea $H$ el subgrupo de $G$ generado por $\left\{x_{\alpha}: \alpha<\lambda\right\}$. 
Entonces $|H|=\lambda$ y por lo tanto $i b(H) \leq \lambda$. Supongamos que $i b(H)=\mu<\lambda$ y tomemos una vecindad simétrica $V$ de la identidad de $G$ tal que $V^{2} \subseteq U$. Entonces existe $F \subseteq H$ con $|F| \leq \mu$ y tal que $H=F \cdot(V \cap H)$. Por lo tanto, para cada $\alpha<\lambda$, existen $g_{\alpha} \in F$ y $v_{\alpha} \in V$ tales que $x_{\alpha}=g_{\alpha} \cdot v_{\alpha}$. Como $|F|<\lambda$, existen $\beta<\alpha<\lambda$ tales que $g_{\beta}=g_{\alpha}=g$. Por lo tanto $x_{\beta}=g \cdot v_{\beta} \mathrm{y}$ $x_{\alpha}=g \cdot v_{\alpha}$. Esto implica que $g=x_{\beta} \cdot v_{\beta}^{-1}$ y, en consecuencia,

$$
x_{\alpha}=x_{\beta} \cdot v_{\beta}^{-1} \cdot v_{\alpha} \in x_{\beta} \cdot V^{-1} \cdot V \subseteq x_{\beta} \cdot V^{2} \subseteq x_{\beta} \cdot U .
$$

Esto contradice la manera en la que la sucesión $\left\{x_{\alpha}: \alpha<\lambda\right\}$ fue construida e implica que $i b(H)=|H|=\lambda$.

Ahora vamos a considerar a un grupo topológico que se expresa como la unión de una cadena creciente de subgrupos. Los siguientes dos resultados analizan cómo se comporta el índice de acotación del grupo en términos de los índices de acotación de los sumandos y de la longitud de la cadena.

LEMA 3.4. Sea $G$ un grupo topológico, $G=\bigcup\left\{G_{\alpha}: \alpha \in \kappa\right\}$, donde $G_{\alpha} \leqslant$ $G_{\beta}<G$ si $\alpha<\beta<\kappa$ y $i b\left(G_{\alpha}\right) \leq \lambda$ para toda $\alpha \in \kappa$. Entonces $i b(G) \leq \lambda \cdot \kappa$.

Demostración. Sea $U$ cualquier vecindad abierta de la identidad de $G$ y, para cada $\alpha \in \kappa$, sea $K_{\alpha} \subseteq G_{\alpha}$ tal que $\left|K_{\alpha}\right| \leq \lambda$ y $K_{\alpha} \cdot\left(U \cap G_{\alpha}\right)=G_{\alpha}$. Entonces, claramente $K=\bigcup\left\{K_{\alpha}: \alpha \in \kappa\right\}$ tiene cardinalidad a lo sumo $\lambda \cdot \kappa$ y $G=K \cdot U$.

TEOREMA 3.5. Si un grupo topológico $G$ es la unión de una cadena creciente de subgrupos, cada uno con índice de acotación estrictamente menor que $\lambda$ para alguin cardinal infinito $\lambda$, entonces $i b(G) \leq \lambda$.

Demostración. Sea $G=\bigcup\left\{G_{\alpha}: \alpha \in \kappa\right\}, G_{\alpha} \leqslant G_{\beta}<G$ si $\alpha \leq \beta<\kappa$, con $i b\left(G_{\alpha}\right)<\lambda$ para toda $\alpha \in \kappa$. Sin pérdida de generalidad podemos suponer que $\kappa$ es regular. Si $\kappa \leq \lambda$ entonces, por el lema 3.4, tenemos que $i b(G) \leq \lambda \cdot \kappa=\lambda$. Supongamos que $\kappa>\lambda$. Si $i b(G)>\lambda$, entonces, por la proposición 3.3, existe un subgrupo $H<G$ tal que $|H|=i b(H)=\lambda$. Como $\kappa>\lambda$, existe $\alpha<\kappa$ con $H \subseteq G_{\alpha}$. Resulta que $\lambda=i b(H) \leq i b\left(G_{\alpha}\right)<\lambda$, lo cual es una contradicción. Concluimos que $i b(G) \leq \lambda$.

Los siguientes dos resultados proporcionan cotas para el peso de grupos topológicos que son uniones de cadenas crecientes de subgrupos con índice de acotación y carácter acotados por un cardinal cualquiera. Dado que todo grupo topológico $G$ satisface la igualdad $w(G)=i b(G) \cdot \chi(G)$ (ver el teorema 1.19), el primero de ellos es una consecuencia inmediata de un resultado de Mikhail Tkachenko en [23].

TEOREMA 3.6. Sea $G=\cup\left\{G_{\alpha}: \alpha \in \kappa\right\}$ un grupo topológico arbitrario; donde $G_{\alpha} \leqslant G_{\beta} \leqslant G$ si $\alpha<\beta<\kappa$. Si $i b\left(G_{\alpha}\right) \cdot \chi\left(G_{\alpha}\right)<\lambda$ para cada $\alpha<\kappa$, entonces $w(G) \leq 2^{\lambda}$. 
DEMOSTRACión. Otra vez podemos suponer, sin pérdida de generalidad, que $\kappa$ es regular. Sabemos que $w\left(G_{\alpha}\right)=i b\left(G_{\alpha}\right) \cdot \chi\left(G_{\alpha}\right)<\lambda$ para cada $\alpha \in \kappa$ (ver el teorema 1.19). El teorema 1.14 garantiza que todo grupo topológico es regular y por lo tanto podemos aplicar el teorema 1.30 para concluir que $w(G) \leq 2^{\lambda}$.

TEOREMA 3.7. Dado un grupo topológico $G$, supongamos que $G=\bigcup\left\{G_{\alpha}\right.$ : $\alpha \in \kappa\}$, donde $G_{\alpha} \leqslant G_{\beta}<G$ si $\alpha<\beta<\kappa$. Si $w\left(G_{\alpha}\right) \leq \lambda$ para toda $\alpha<\kappa y$ $c f(\kappa) \neq \lambda^{+}$, entonces $w(G) \leq 2^{\lambda}$.

Demostración. Podemos suponer que $\kappa$ es regular y que $\kappa \neq \lambda^{+}$.

Caso 1. $\kappa \leq \lambda$. Sabemos que $n w\left(G_{\alpha}\right) \leq w\left(G_{\alpha}\right)=\leq \lambda$ para toda $\alpha<\kappa$, así que, por el teorema $1.24, n w(G) \leq \kappa \cdot \lambda=\lambda$. Además sabemos que $d(G) \leq$ $n w(G)$ (ver el inciso (a) del teorema 1.2). Como $G$ es regular, podemos aplicar el teorema 1.3 para concluir que $w(G) \leq 2^{d(G)} \leq 2^{n w(G)} \leq 2^{\lambda}$.

Caso 2. $\kappa>\lambda^{+}$. En este caso $w\left(G_{\alpha}\right) \leq \lambda<\lambda^{+}$para todo $\alpha<\kappa$. Sea $H$ cualquier subespacio de $G$ de cardinalidad a lo sumo $\lambda^{+}$. Como $\kappa$ es regular y $\kappa>\lambda^{+}$, existe $\alpha<\kappa$ tal que $H \subseteq G_{\alpha}$. Por lo tanto $w(H) \leq w\left(G_{\alpha}\right)<\lambda^{+}$. Hemos probado que todo subespacio de $G$ de cardinalidad $\leq \lambda^{+}$tiene peso $<\lambda^{+}$. Esto hace posible aplicar el teorema 1.28 y concluir que $w(G)<\lambda^{+} \leq$ $2^{\lambda}$.

El siguiente ejemplo muestra que la hipótesis $c f(\kappa) \neq \lambda^{+}$en el teorema 3.7 es esencial. Vamos a construir un grupo topológico $G$ de peso $\geq 2^{\omega_{1}}$ que se puede expresar como la unión de una cadena creciente de longitud $\omega_{1}$ de subgrupos, cada uno de ellos primero numerable y $\aleph_{0}$-acotado. Si se supone la Hipótesis del Continuo, entonces $2^{\omega_{1}}>2^{\omega}$ y en consecuencia el teorema 3.7 no se cumple para $\lambda=\omega$ y $\kappa=\omega_{1}$. Como la Hipótesis del Continuo es consistente con los axiomas usuales de la teoría de conjuntos, el ejemplo establece que el teorema 3.7 no se puede demostrar sin la hipótesis acerca de la cofinalidad de la longitud de la cadena.

Recordemos que si $\mathcal{U}$ es un ultrafiltro y $\mathcal{A} \subseteq U$, entonces decimos que la familia $\mathcal{A}$ es base de $\mathcal{U}$ si $U$ es el único ultrafiltro que extiende a $\mathcal{A}$, en otras palabras, si para todo $U \in U$, existe $A \in \mathcal{A}$ tal que $A \subseteq U$. El carácter de $U$ es la mínima cardinalidad de una base de $U$ y se denota $\chi(\mathcal{U})$. Por un resultado de Kunen (ver [20]), para cada cardinal infinito $\kappa$ existe un ultrafiltro uniforme (es decir, que consiste únicamente de conjuntos de cardinalidad $\kappa$ ) y no principal sobre $\kappa$ con carácter $2^{\kappa}$.

EJEMPLO 3.8. Existe un grupo topológico $G$ con $w(G) \geq 2^{\omega_{1}}$ y tal que $G$ se puede expresar como la unión de una cadena de longitud $\omega_{1}$ de subgrupos, cada uno de los cuales es es primero numerable y $\aleph_{0}$-acotado.

DEMOSTRACión. Sea $U$ un ultrafiltro en $\omega_{1}$ con carácter $2^{\omega_{1}}$ y tal que todo elemento de $\mathcal{U}$ tiene cardinalidad $\omega_{1}$. Sea $G$ el conjunto de todos los 
subconjuntos finitos de $\omega_{1}$. Si hacemos $A \Delta B=(A \backslash B) \cup(B \backslash A)$ para cualesquiera $A, B \in G$, entonces $(G, \triangle)$ es un grupo Booleano (es decir, de orden 2) con el conjunto vacío, $\emptyset$, como la identidad.

Ahora bien, para cada $A \in G$ y cada $F \in \mathcal{U}$, sea $U_{F}^{A}=\{A \triangle S: S \subseteq$ $F,|S|<\omega\}$. Entonces la familia $\mathcal{B}=\left\{U_{F}^{A}: A \in G, F \in \mathcal{U}\right\}$ es base para una topología de grupo $\tau$ en $G$. Es fácil ver que para todo $A \in G, A \in U_{F}^{A}$ para cualquier $F \in U$, pues $A=A \triangle \emptyset$. Además, si $C \in U_{E}^{A} \cap U_{F}^{B}$, donde $A, B \in G$ y $E, F \in U$, se puede verificar fácilmente que $C \in U_{E \cap F}^{C} \subseteq U_{E}^{A} \cap U_{F}^{B}$. Con esto queda demostrado que la familia $\mathcal{B}=\left\{U_{F}^{A}: A \in G, F \in \mathcal{U}\right\}$ es base para una topología en $G$. Falta ver que las operaciones de grupo son continuas. Dado que el inverso de $A \in G$ es él mismo, el inverso es una función continua. Para comprobar que la función $\triangle: G \times G \longrightarrow G$ es continua, basta verificar que para cualesquiera $A, B \in G$ y cualquier vecindad $U_{F}^{A \Delta B}$ de $A \triangle B$, se tiene que $U_{F}^{A} \triangle U_{F}^{B} \subseteq U_{F}^{A \Delta B}$.

Así, $(G, \Delta, \tau)$ es un grupo topológico de cardinalidad $\omega_{1}$ y por lo tanto se puede expresar como la unión de una cadena creciente de subgrupos numerables, $G=\bigcup\left\{G_{\alpha}: \alpha \in \omega_{1}\right\}$ haciendo $G_{0}=\{\emptyset\}$ y definiendo, para cada $\alpha \in \omega_{1}, G_{\alpha}$ como el subgrupo de $G$ generado por $\cup\left\{G_{\beta}: \beta \in \alpha\right\} \cup\left\{p_{\alpha}\right\}$, donde $p_{\alpha} \notin \cup\left\{G_{\beta}\right.$ : $\beta \in \alpha\}$. Afirmamos que, aún cuando $w(G) \geq 2^{\omega_{1}}, i b\left(G_{\alpha}\right) \cdot \chi\left(G_{\alpha}\right) \leq \omega$ para toda $\alpha \in \omega_{1}$. En efecto, $i b\left(G_{\alpha}\right) \leq \omega$ para toda $\alpha \in \omega_{1}$ porque todos ellos son grupos numerables. Para ver que $\chi\left(G_{\alpha}\right) \leq \omega$ para toda $\alpha \in \omega_{1}$, consideremos a la identidad de $G$, el conjunto vacío $\emptyset$. Para todo conjunto $S, \emptyset S=S$, por lo que, dado cualquier $F \in U, U_{F}^{\natural}=\{S \subset F:|S|<\omega\}$. Note que para toda $\alpha \in \omega_{1}, G_{\alpha}$ es una familia numerable de subconjuntos finitos de $\omega_{1}$ y por lo tanto $\cup G_{\alpha}$ es numerable. Tomemos $F \in \mathcal{U}$ tal que $F \cap \cup G_{\alpha}=\emptyset$. Entonces $U_{F}^{\emptyset} \cap G_{\alpha}=\{\emptyset\}$, ya que si no fuera así existiría un conjunto finito $S \subseteq F$ no vacío tal que $S \in G_{\alpha}$, contradiciendo nuestra elección de $F$. Por lo tanto $G_{\alpha}$ es un grupo discreto y tiene, por lo tanto, carácter numerable. Finalmente, probamos que $w(G) \geq \chi(U)=2^{\omega_{1}}$. Para ver esto será suficiente con demostrar que el carácter de $G$ en la identidad es $\geq \chi(\mathcal{U})$. Consideremos cualquier base local para la identidad de $G, \mathcal{B}=\left\{U_{\alpha}: \alpha \in \kappa\right\}$, donde, para cada $\alpha \in \kappa, U_{\alpha}=U_{F_{\alpha}}^{6}$ para algún $F_{\alpha} \in \mathcal{U}$. Sea $F \in U$. Entonces $\emptyset \in U_{F}^{\emptyset}$ y por lo tanto existe $\alpha \in \kappa$ tal que $U_{\alpha} \subseteq U_{F}^{\varphi}$. Esto implica que para cualquier conjunto finito $T \subseteq F_{\alpha}$, $T=\emptyset T \subseteq F$. Por lo tanto $F_{\alpha} \subseteq F$, y concluimos que $\kappa \geq \chi(\mathcal{U})=2^{\omega_{1}}$. Con esto queda probado que $w(G) \geq 2^{\omega_{1}}$.

\section{Cotas para la cardinalidad de los grupos}

Es bien sabido que todo grupo topológico $G$ satisface que $|G| \leq 2^{i b(G) \cdot \psi(G)}$ (ver el teorema 1.23). En esta sección probamos una versión de este resultado en términos de cadenas crecientes cuando el grupo $G$ es numerablemente compacto y damos un ejemplo que muestra que el resultado no es válido en general, ni siquiera para grupos pseudocompactos. 
TEOREMA 3.9. Si $G$ es un grupo topológico numerablemente compacto, $G=$ $\bigcup\left\{G_{\alpha}: \alpha \in \kappa\right\}$, donde $G_{\alpha} \leqslant G_{\beta}<G$ si $\alpha<\beta<\kappa$ y $\psi\left(G_{\alpha}\right) \leq \omega$ para toda $\alpha \in \kappa$, entonces $|G| \leq 2^{\omega}$.

Demostración. Observemos primero que $G$ es totalmente acotado. En efecto, si no lo es, tomando cualquier vecindad $U$ de la identidad en $G$ tal que $K \cdot U \neq G$ para todo subconjunto finito $K$ de $G$, se puede construir, de manera análoga a la demostración de la proposición 3.3, una sucesión infinita de puntos de $G$ que no tiene puntos de acumulación. Así que para cada $\alpha<\kappa$, tenemos que $i b\left(G_{\alpha}\right) \leq \omega$. Por lo tanto $\left|G_{\alpha}\right| \leq 2^{i b\left(G_{\alpha}\right) \cdot \psi\left(G_{\alpha}\right)} \leq 2^{\omega}$ para toda $\alpha \in \kappa$ (ver el teorema 1.23).

Si $\kappa \leq 2^{\omega}$, entonces $|G| \leq \kappa \cdot 2^{\omega} \leq 2^{\omega}$. Consideremos, entonces, el caso $\kappa>2^{\omega}$. Sea $\alpha<\kappa$ arbitrario. Vamos a definir por recursión, a la manera de Comfort y Saks (ver [7]), una sucesión creciente $\left\{H_{\xi}: \xi \in \omega_{1}\right\}$ de subgrupos de $G$ de cardinalidad $\leq 2^{\omega}$ tales que $G_{\alpha} \leqslant H_{\xi}$ para todo $\xi \in \omega_{1}$. Hagamos $H_{0}=G_{\alpha}$, fijemos $\xi \in \omega_{1}$ y supongamos $H_{\beta}$ definido para toda $\beta \in \xi$. Si $\xi$ es un ordinal límite, el grupo $H_{\xi}=\bigcup\left\{H_{\beta}: \beta \in \xi\right\}$, tiene cardinalidad $\leq 2^{\omega}$ porque $\xi<\omega_{1}$ y cada $H_{\beta}$ tiene cardinalidad $\leq 2^{\omega}$. Si $\xi$ es un ordinal sucesor, digamos $\xi=\beta+1$, entonces sea $\left\{S_{\nu}: \nu \in A\right\}$ una enumeración de todos los subconjuntos numerables de $H_{\beta}$ que no tienen un punto de acumulación en $H_{\beta}$. Note que $|A| \leq 2^{\omega}$ pues por hipótesis $\left|H_{\beta}\right| \leq 2^{\omega}$. Para cada $\nu \in A$, sea $p_{\nu}$ un punto de acumulación de $S_{\nu}$ en $G$ y sea $F_{\xi}=H_{\beta} \cup\left\{p_{\nu}: \nu \in A\right\}$. Entonces podemos definir a $H_{\xi}$ como el subgrupo de $G$ generado por $F_{\xi}$. Si hacemos $K_{\alpha}=\bigcup\left\{H_{\xi}: \xi \in \omega_{1}\right\}$, entonces es claro que $G_{\alpha}<K_{\alpha}<G$, que $K_{\alpha}$ es numerablemente compacto y que $\left|K_{\alpha}\right| \leq 2^{\omega}$. Ahora bien, como $\kappa=c f(\kappa)>2^{\omega}$, existe $\alpha^{\prime} \in \kappa$ tal que $K_{\alpha} \subseteq G_{\alpha^{\prime}}$ y por lo tanto $\psi\left(K_{\alpha}\right) \leq \psi\left(G_{\alpha^{\prime}}\right) \leq \omega$.

Hemos demostrado que para cada $\alpha<\kappa$ existe un grupo numerablemente compacto $K_{\alpha}$ tal que $G_{\alpha} \leqslant K_{\alpha} \leqslant G_{\alpha^{\prime}}$ para algún ordinal $\alpha^{\prime}>\alpha$. Como $K_{\alpha}$ es numerablemente compacto y $\psi\left(K_{\alpha}\right) \leq \omega$, tenemos que $\chi\left(K_{\alpha}\right) \leq \omega$. Aplicando el teorema 1.19 , obtenemos

$$
w\left(K_{\alpha}\right)=i b\left(K_{\alpha}\right) \cdot \chi\left(K_{\alpha}\right) \leq \omega
$$

para toda $\alpha<\kappa$. Pero $G_{\alpha} \subseteq K_{\alpha}$, lo cual implica que $w\left(G_{\alpha}\right) \leq \omega$ para toda $\alpha<\kappa$.

Dado que todo $X \subseteq G$ con cardinalidad a lo sumo $\omega_{1}$ está contenido en algún $G_{\alpha}$ y tiene, por lo tanto, peso numerable, concluimos, aplicando el teorema 1.28, que $w(G) \leq \omega$. Resulta que en este caso, en virtud del teorema 1.1, también $|G| \leq 2^{\omega}$.

En el teorema anterior la topología de grupo jugó un papel muy importante. Observemos que este resultado no es válido en general para espacios numerablemente compactos. Para ver esto, considere cualquier cardinal $\kappa>2^{\omega}$ y tome a $X$ como el conjunto de todos los ordinales menores que $\kappa$ con cofinalidad numerable. Entonces $X$ con la topología inducida por el orden es un 
espacio numerablemente compacto con pseudocarácter numerable. Sin embargo $|X|>2^{\omega}$.

El teorema 3.9 tampoco es válido para grupos pseudocompactos. Para demostrar esto en el siguiente ejemplo construimos un grupo topológico pseudocompacto $G$ que se puede expresar como la unión de una cadena creciente de subgrupos (que serán totalmente acotados, siendo subgrupos de un grupo topológico totalmente acotado), cada uno de pseudocarácter numerable, y tal que $|G|=\mathfrak{c}^{+}$, donde $\mathfrak{c}$ es la cardinalidad del continuo. Recordemos que un grupo Booleano es un grupo cuyos elementos son todos de orden 2.

EJEMPLO 3.10. Existe un grupo topológico pseudocompacto de cardinalidad $c^{+}$que se puede expresar como la unión de una cadena creciente de subgrupos, todos ellos de pseudocarácter numerable.

DEMostración. Sea $D$ el grupo $\{0,1\}$ con la topología discreta y sea $H=\left(D^{\omega}\right)^{c^{+}}$. Vamos a definir un subgrupo denso pseudocompacto $G \leqslant H$. Tomemos cualquier grupo Booleano $\Sigma$ de cardinalidad $c^{+}$y enumerémoslo de la siguiente manera:

$$
\Sigma=\left\{x_{\alpha}: \alpha<c^{+}\right\} .
$$

Para cada conjunto $S \subseteq \mathrm{c}^{+}$, hagamos $H_{S}=\left(D^{\omega}\right)^{S}$ y pongamos $\mathcal{A}=\cup\left\{H_{S}\right.$ : $\left.S \subseteq \mathrm{c}^{+},|S| \leq \omega\right\}$. Note que si $S$ es un subconjunto numerable de $\mathrm{c}^{+},\left|H_{S}\right| \leq$ $\left(2^{\omega}\right)^{\omega}=\mathfrak{c}$, por lo que $|\mathcal{A}| \leq \mathfrak{c} \cdot\left(\mathfrak{c}^{+}\right)^{\omega}=\mathfrak{c}^{+}$. Podemos, por lo tanto, escribir a $\mathcal{A}$ de la manera siguiente:

$$
\mathcal{A}=\left\{q_{\alpha}: \alpha<\mathrm{c}^{+}\right\} .
$$

Vamos a definir recursivamente una familia $\left\{\widetilde{f}_{\xi}: \xi<c^{+}\right\}$de homomorfismos de grupo de $\Sigma$ en $D^{\omega}$. Para poder hacer esto, definiremos primero, para cada $\alpha<c^{+}$, un subconjunto $P_{\alpha} \subseteq \mathrm{c}^{+}$y una familia de homomorfismos $\left\{f_{\xi, \alpha}\right.$ : $\left.X_{\xi, \alpha} \longrightarrow D^{\omega}\right\}_{\xi \in P_{\alpha}}$ de tal suerte que se satisfagan las siguientes condiciones:

(i) $P_{\alpha}$ es un subconjunto no vacío de $\mathrm{c}^{+}$y $\left|P_{\alpha}\right| \leq \mathrm{c}$;

(ii) $X_{\xi, \alpha}<\Sigma$ y $\left|X_{\xi, \alpha}\right| \leq \mathrm{c}$ para cada $\xi \in P_{\alpha}$;

(iii) $P_{\beta} \subseteq P_{\alpha}$ si $\beta \leq \alpha$;

(iv) para cada $\xi \in P_{\beta}, X_{\xi, \beta} \subseteq X_{\xi, \alpha}$ si $\beta \leq \alpha$;

(v) para cada $\xi \in P_{\beta}, f_{\xi, \alpha} \mid X_{\xi, \beta}=f_{\xi, \beta}$ si $\beta \leq \alpha$.

Fijemos $\alpha<c^{+}$y supongamos que $P_{\beta}$ y $\left\{f_{\xi, \beta}: X_{\xi, \beta} \longrightarrow D^{\omega}\right\}_{\xi \in P_{\beta}}$ han sido definidos para toda $\beta<\alpha$. Si $\alpha$ es un ordinal límite, hagamos $P_{\alpha}=\bigcup\left\{P_{\beta}\right.$ : $\beta<\alpha\}$ y, para cada $\xi \in P_{\alpha}$,

$$
X_{\xi, \alpha}=\bigcup\left\{X_{\xi, \beta}: \beta<\alpha, \xi \in P_{\beta}\right\}
$$

y

$$
f_{\xi, \alpha}=\bigcup\left\{f_{\xi, \beta}: \beta<\alpha, \xi \in P_{\beta}\right\}
$$


Claramente se satisfacen las condiciones (i) -(v). Supongamos ahora que $\alpha$ es un ordinal sucesor, digamos que $\alpha=\beta+1$. La construcción consta de varias etapas. En la primera etapa nos aseguramos de que $x_{\beta} \in X_{\xi, \alpha}$, para que cualquier elemento de $\Sigma$ esté en el dominio de alguna función $f_{\xi, \alpha}$. Las etapas 2 y 3 garantizan que el subgrupo resultante de $H$ sea pseudocompacto. Finalmente, la etapa 4 sirve para expresar al grupo resultante $G$ como unión de una cadena creciente de subgrupos de pseudocarácter numerable. Se usará repetidamente el hecho conocido de que si $X$ es un subgrupo de un grupo booleano $Y$, entonces todo homomorfismo de $X$ a $D$ se puede extender a $Y$.

Paso 1. Consideremos un $\xi \in P_{\beta}$ arbitrario. Sea $Y_{\xi, \alpha}=\left\langle X_{\xi, \beta} \cup\left\{x_{\beta}\right\}\right\rangle<\Sigma$. Por hipótesis de inducción, el homomorfismo $f_{\xi, \beta}: X_{\xi, \beta} \longrightarrow D^{\omega}$ ya ha sido definido, así que, para toda $n \in \omega, \pi_{n} \circ f_{\xi, \beta}: X_{\xi, \beta} \longrightarrow D$ es un homomorfismo, donde $\pi_{n}: D^{\omega} \longrightarrow D$ es la proyección natural. Para cada $n \in \omega$, sea $h_{n}$ : $Y_{\xi, \alpha} \longrightarrow D$ una extensión de $\pi_{n} \circ f_{\xi, \beta}$. Entonces el producto diagonal

$$
g_{\xi, \alpha}=\triangle_{n \in \omega} h_{n}: Y_{\xi, \alpha} \longrightarrow D^{\omega}
$$

es una extensión de $f_{\xi, \beta}$ to $Y_{\xi, \alpha}$, es decir, $g_{\xi, \alpha} \mid X_{\xi, \beta}=f_{\xi, \beta}$. Hemos definido, para cada $\xi \in P_{\beta}$, un subgrupo $Y_{\xi, \alpha}<\Sigma$ y un homomorfismo $g_{\xi, \alpha}$ de tal forma que $X_{\xi, \beta} \leqslant Y_{\xi, \alpha}<\Sigma ;\left|Y_{\xi, \alpha}\right| \leq c ; x_{\beta} \in Y_{\xi, \alpha} ; g_{\xi, \alpha}: Y_{\xi, \alpha} \longrightarrow D^{\omega}$ y $g_{\xi, \alpha} \mid X_{\xi, \beta}=f_{\xi, \beta}$.

Paso 2. Ahora consideremos $q_{\beta} \in \mathcal{A}$. Entonces, por definición de $\mathcal{A}$, existe un conjunto numerable $S$ de $c^{+}$tal que $q_{\beta} \in H_{S}$, es decir, $q_{\beta} \in\left(D^{\omega}\right)^{S}$. Sea $K$ el subgrupo de $\Sigma$ generado por $\bigcup\left\{Y_{\xi, \alpha}: \xi \in P_{\beta} \cap S\right\}$. Entonces, en vista de que $\left|P_{\beta}\right| \leq \mathrm{c}$ y para cada $\xi \in P_{\beta},\left|Y_{\xi, \alpha}\right| \leq \mathfrak{c}$, se tiene que $|K| \leq \mathfrak{c}$ y podemos, por tanto, encontrar $x \in \Sigma \backslash K$. Para cada $\xi \in P_{\beta} \cap S$, sea

$$
Z_{\xi, \alpha}=\left\langle Y_{\xi, \alpha} \cup\{x\}\right\rangle=Y_{\xi, \alpha}+\{0, x\}<\Sigma .
$$

Para cada $\xi \in P_{\beta} \cap S$ hemos definido $g_{\xi, \alpha}: Y_{\xi, \alpha} \longrightarrow D^{\omega}$, de tal forma que, para cada $n \in \omega, \pi_{n} \circ g_{\xi, \alpha}: Y_{\xi, \alpha} \longrightarrow D$ es un homomorfismo. Como $x \notin K$, podemos definir, para cada $n$, un homomorfismo $j_{n}: Z_{\xi, \alpha} \longrightarrow D$ tal que $j_{n}(x)=q_{\beta}(\xi)(n)$ y $j_{n} \mid Y_{\xi, \alpha}=\pi_{n} \circ g_{\xi, \alpha}$. Entonces, definiendo $h_{\xi, \alpha}$ como el producto diagonal de la familia $\left\{j_{n}: n \in \omega\right\}$ obtenemos, para cada $\xi \in P_{\beta} \cap S$, un subgrupo $Z_{\xi, \alpha}$ de $\Sigma$ y un homomorfismo $h_{\xi, \alpha}$ que satisfacen las siguientes condiciones: $Y_{\xi, \alpha}<Z_{\xi, \alpha}<\Sigma ;\left|Z_{\xi, \alpha}\right| \leq c ; h_{\xi, \alpha}: Z_{\xi, \alpha} \longrightarrow D^{\omega} ; h_{\xi, \alpha} \mid Y_{\xi, \alpha}=g_{\xi, \alpha} \mathrm{y}$

$$
h_{\xi, \alpha}(x)=q_{\beta}(\xi) \text {. }
$$

Paso 3. Si, por otro lado, $\xi \in S \backslash P_{\beta}$, denótese por $V_{\xi, \alpha}$ el subgrupo de $\Sigma$ generado por $x$ y defínase $j(x)=q_{\beta}(\xi)$. Entonces $j$ se puede extender a un homomorfismo $l_{\xi, \alpha}: V_{\xi, \alpha} \longrightarrow D^{\omega}$, así que tenemos, en este caso: $V_{\xi, \alpha}<\Sigma$; 
$\left|V_{\xi, \alpha}\right| \leq \mathfrak{c} ; l_{\xi, \alpha}: V_{\xi, \alpha} \longrightarrow D^{\omega} \mathrm{y}$

$$
l_{\xi, \alpha}(x)=q_{\beta}(\xi)
$$

Paso 4. Escojamos ahora $\xi_{\alpha} \in \mathrm{c}^{+} \backslash\left(P_{\beta} \cup S\right)$; esto es posible porque $\left|P_{\beta}\right| \leq \mathrm{c}$ y $|S| \leq \aleph_{0}$. Considérese

$$
X_{\alpha}=\left\langle\left\{x_{\nu}: \nu<\alpha\right\}\right\rangle<\Sigma .
$$

Si $\alpha \geq c$, sea $W_{\xi_{\alpha}, \alpha}=X_{\alpha}$, si no, sea $W_{\xi_{\alpha}, \alpha}=X_{c}=\left\langle\left\{x_{\nu}: \nu<c\right\}\right\rangle$. Entonces $W_{\xi_{\alpha}, \alpha}$ es un grupo booleano de cardinalidad $\mathrm{c} y$, por lo tanto, algebraicamente isomorfo a $D^{\omega}$. Sea $k_{\xi_{\alpha}, \alpha}: W_{\xi_{\alpha}, \alpha} \longrightarrow D^{\omega}$ un isomorfismo. Tenemos la siguiente situación: $W_{\xi_{\alpha}, \alpha}<\Sigma ;\left|W_{\xi_{\alpha}, \alpha}\right| \leq \mathfrak{c} ; X_{\alpha} \subseteq W_{\xi_{\alpha}, \alpha} \mathrm{y}$

$$
k_{\xi_{\alpha}, \alpha}: W_{\xi_{\alpha}, \alpha} \longrightarrow D^{\omega} \text { es un isomorfismo. }
$$

Finalmente estamos en posición de definir $P_{\alpha}$ y $f_{\xi, \alpha}$ para todo $\xi \in P_{\alpha}$. Hagamos $P_{\alpha}=P_{\beta} \cup S \cup\left\{\xi_{\alpha}\right\}$ y, para cada $\xi \in P_{\alpha}$, definamos $X_{\xi, \alpha}$ y $f_{\xi, \alpha}$ como sigue:

(a) Si $\xi \in P_{\beta} \backslash S, X_{\xi, \alpha}=Y_{\xi, \alpha}$ y $f_{\xi, \alpha}=g_{\xi, \alpha}$ (ver el paso 1);

(b) si $\xi \in P_{\beta} \cap S, X_{\xi, \alpha}=Z_{\xi, \alpha}$ y $f_{\xi, \alpha}=h_{\xi, \alpha}$ (ver el paso 2);

(c) si $\xi \in S \backslash P_{\beta}, X_{\xi, \alpha}=V_{\xi, \alpha}$ y $f_{\xi, \alpha}=l_{\xi, \alpha}$ (ver el paso 3);

(d) si $\xi=\xi_{\alpha}, X_{\xi, \alpha}=W_{\xi_{\alpha}, \alpha}$ y $f_{\xi, \alpha}=k_{\xi \alpha, \alpha}$ (ver el paso 4).

Es fácil ver que se satisfacen las condiciones (i)-(v). Note también que, para cada subconjunto numerable $S \subseteq \mathrm{c}^{+}$, todo elemento $q_{\beta} \in H_{S}$ fue considerado en los pasos 2 y 3 de la construcción, por lo que $U\left\{P_{\alpha}: \alpha<c^{+}\right\}=c^{+}$. Para cada $\xi<c^{+}$sea $X^{\xi}=\bigcup\left\{X_{\xi, \alpha}: \alpha<c^{+}, \xi \in P_{\alpha}\right\}$. Entonces $X^{\xi}<\Sigma$ y, dado que $X_{\xi, \beta} \subseteq X_{\xi, \alpha}$ y $f_{\xi, \beta} \subseteq f_{\xi, \alpha}$ para $\beta \leq \alpha, f_{\xi}=\bigcup\left\{f_{\xi, \alpha}: \alpha<c^{+}\right\}: X^{\xi} \longrightarrow D^{\omega}$ es un homomorfismo. Se sigue que para $n \in \omega$, el homomorfismo $\pi_{n} \circ f_{\xi}: X^{\xi} \longrightarrow D$ se puede extender a $f_{\xi}^{n}: \Sigma \longrightarrow D$. Haciendo

$$
\widetilde{f_{\xi}}=\triangle_{n \in \omega} f_{\xi}^{n}: \Sigma \longrightarrow D^{\omega}
$$

obtenemos, para cada $\xi \in \mathrm{c}^{+}$, un homomorfismo $\widetilde{f}_{\xi}$ de $\Sigma$ en $D^{\omega}$ con $\widetilde{f}_{\xi}\left\lceil X^{\xi}=\right.$ $f_{\xi}$. Sea $\phi$ el producto diagonal de las $\tilde{f}_{\xi}$ 's:

$$
\phi=\Delta_{\xi \in c^{+}} \widetilde{f_{\xi}}: \Sigma \longrightarrow\left(D^{\omega}\right)^{c^{+}}
$$

y definamos a $G=\phi(\Sigma)<H$. Probamos ahora que $G$ es un subgrupo denso y pseudocompacto de $H$, de cardinalidad $\mathrm{c}^{+}$, y que puede ser escrito como la unión de una cadena creciente de subgrupos, cada uno de pseudocarácter numerable. Como $H$ es un grupo compacto, todos sus subgrupos son totalmente acotados. Para ver que $G$ es un subgrupo denso pseudocompacto de $H$ basta mostrar que para todo subconjunto numerable $S \subseteq \mathrm{c}^{+}, \pi_{S}(G)=\left(D^{\omega}\right)^{S}$, donde $\pi_{S}$ es 
la proyección. Tomemos pues cualquier subconjunto numerable $S$ de $c^{+}$y un punto $y \in\left(D^{\omega}\right)^{S}$ arbitrario; entonces $y \in \mathcal{A}$ y por lo tanto $y=q_{\beta}$ para alguna $\beta<\mathrm{c}^{+}$. En el $\beta+1$-ésimo paso inductivo de nuestra construcción definimos $x \in \Sigma$ de tal forma que $f_{\xi, \beta+1}(x)=q_{\beta}(\xi)=y(\xi)$ para cualesquiera $\xi \in S$ (ver $3.1,3.2$, (b) y (c)). En consecuencia $x \in X^{\xi}$ y $\widetilde{f}_{\xi}(x)=f_{\xi}(x)=f_{\xi, \beta+1}(x)=y(\xi)$ para todo $\xi \in S$. Concluimos que $\pi_{S}(\phi(x))=y$ y por lo tanto $\pi_{S}(G)=\left(D^{\omega}\right)^{S}$. Para ver que $|G|=\mathbf{c}^{+}$basta observar que dados cualesquiera dos elementos distintos de $\Sigma$, digamos $x_{\alpha}$ y $x_{\beta}$, con $\alpha>\beta$, en el $\alpha+1$-ésimo paso de nuestra construcción definimos un ordinal $\xi_{\alpha+1}$ tal que $f_{\xi \alpha+1, \alpha+1}\left(x_{\beta}\right) \neq f_{\xi \alpha+1, \alpha+1}\left(x_{\alpha}\right)$ (ver 3.3, y (d)), y por lo tanto $f_{\xi \alpha+1}\left(x_{\beta}\right) \neq f_{\xi \alpha+1}\left(x_{\alpha}\right)$, de donde se sigue que $\phi$ es inyectiva. Finalmente, para cada $\alpha<c^{+} \operatorname{sean} X_{\alpha}=\left\langle\left\{x_{\nu}: \nu<\alpha\right\}\right\rangle$ y $G_{\alpha}=\phi\left(X_{\alpha}\right)$. Entonces $\Sigma=\bigcup\left\{X_{\alpha}: \alpha<\mathrm{c}^{+}\right\}$y $G=\bigcup\left\{G_{\alpha+1}: \alpha<\mathrm{c}^{+}\right\}$. Falta ver que todos ellos tienen pseudocarácter numerable. Para ver esto, elijamos cualquier elemento $a \in G_{\alpha+1}$ y notemos que existe $x \in X_{\alpha+1}$ tal que $\phi(x)=a$. Sea $\beta=\alpha+1$. En el $\beta$-ésimo paso de nuestra construcción definimos un ordinal $\xi_{\beta}$ tal que $f_{\xi_{\beta}, \beta}: X_{\beta} \longrightarrow D^{\omega}$ es un homomorfismo inyectivo (ver 3.3 y (d)). Así, por definición de $f_{\xi_{\beta}}, f_{\xi_{\beta}} \mid X_{\beta}$ es uno a uno. Sea $\pi_{\xi_{\beta}}:\left(D^{\omega}\right)^{c^{+}} \longrightarrow D^{\omega}$ la proyección. Entonces, en vista de que $\pi_{\xi_{\beta}}(a)=f_{\xi_{\beta}}(x)$, la restricción $\pi_{\xi_{\beta}} \mid G_{\beta}$ : $G_{\beta} \longrightarrow D^{\omega}$ es inyectiva. Por lo tanto $\psi\left(G_{\beta}\right) \leq \psi\left(D^{\omega}\right)=\omega$. 


\section{Bibliografía}

1. P. Alexandroff, Zur Theorie der topologischen Räume, Dokl. Akad. Nauk. SSSR 11 (1936), 55-58 (en ruso).

2. A. V. Arhangel'skii and V.I. Ponomarev, On dyadic compacta, Dokl. AN SSSR 182 (1968), 993-996 (en ruso).

3. G. Birkhoff, A note on topological groups, Compositio Math. 3 (1936), 427-430.

4. J. Cleary and S. A. Morris, Locally dyadic topological groups, Bull. Austral. Math. Soc. 40 (1989), 417-419.

5. W.W. Comfort, Topological groups, en: Handbook of Set-theoretic Topology, K. Kunen and J. E. Vaughan, editores, Capítulo 24, páginas 1143-1263. North-Holland, Amsterdam 1984.

6. W.W. Comfort and K.A. Ross, Pseudocompactness and uniform continuity in topological groups, Pacific J. Math. 16 (1966) 483-496.

7. W.W. Comfort and V. Saks, Countably compact groups and finest totally bounded topologies, Pacific J. Math. 49 (1973) 33-44.

8. A. Dow, An introduction to application of elementary submodels to topology, Topology Proc. 13 (1988), 17-72.

9. B. A. Efimov, Dyadic compacta, Trudy Mosc. Matem. Obshch. 14 (1965), 211-247 (en ruso). Traducción al inglés en: Trans. Mosc. Math. Soc. 14 (1965), 229-267.

10. B. A. Efimov, Mappings and embeddings of dyadic bicompacta, Mat. Sbornik 103 (1977), 52-68 (en ruso).

11. R. Engelking, General Topology, Heldermann Verlag, 1989.

12. A.S. Esenin-Vol'pin, On the relation between the local and integral weight in dyadic bicompacta, Dokl. Akad. Nauk SSSR 68 (1949), 441-444.

13. J. Gerlitz, Continuous functions on products of topological spaces, Fund. Math. 106 (1980), 67-75.

14. I. Guran, On topological groups close to being Lindelöf, Soviet Math. Dokl. 23 (1981), 173-175.

15. O. Hagler, On the structure of $S$ and $C(S)$ for $S$ dyadic, Trans. Amer. Math. Soc. 214 (1975), 415-428.

16. A. Hajnal and I. Juhász, Having small weight is determined by the small subspaces, Proc. Amer. Math. Soc. 79 (1980), no. 4, 657-658.

17. I. Juhász and Z. Szentmiklossy, Increasing strengthenings of cardinal function inequalities, Fund. Math. 126 (1986), 209-216.

18. I. Juhász, Cardinal functions in topology - ten years later, Math. Centre Tracts, Amsterdam, 1980.

19. S. Kakutani, Über die Metrization der topologischen Gruppen, Proc. Imp. Acad. Tokyo 12 (1936), 82-84.

20. K. Kunen, Trans. Am. Math. Soc., 172 (1972), 299.

21. K. Kunen, Set Theory, North Holland, 1980.

22. I. Osuna, Espacios compactos diádicos, Tesis de Maestría, UAM, 1998. 
23. M. G. Tkachenko, The behaviour of cardinal invariants under the union operation for a chain of spaces, Moscow Univ. Math. Bull. 33 (1978), no. 4, 39-46. Original en ruso en: Vestnik Moskov. Univ. Ser. I Mat. Mekh. no. 4 (1978), 50-58.

24. M. G. Tkachenko, Chains and cardinals, Soviet Math. Dokl. 19 (1978), no. 2, 382-385.

25. M. G. Tkachenko, The Souslin property in free topological groups over compact spaces, Math. Notes 34 (1983), 790-793.

26. M. G. Tkachenko, Sum theorems for the tightness and $\pi$-character in the class of compact spaces, Comment. Math. Univ. Carolin. 24 (1983), no. 1, 51-62.

27. M. G. Tkachenko, Introduction to topological groups, Topology Appl. 86 (1998), 179-231.

28. M.G. Tkachenko et al., Grupos topológicos, UAM-Iztapalapa, México, 1998.

29. A. Weil, La mésure invariante dans les éspaces de groupes et les éspaces homogènes, Enseignement Math. 35 (1936), 241. 